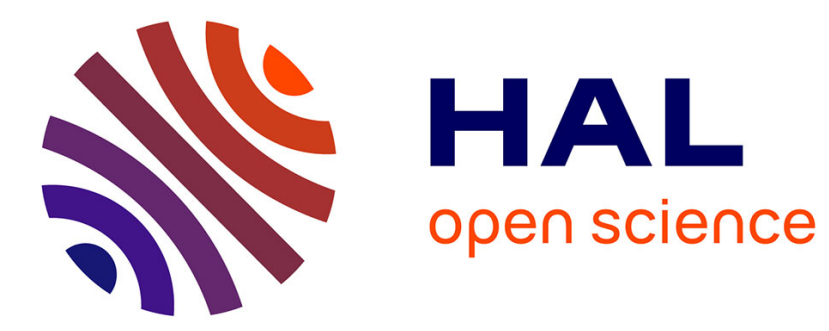

\title{
Precessional instability of a fluid cylinder
}

Romain Lagrange, Patrice Meunier, François Nadal, Christophe Eloy

\section{To cite this version:}

Romain Lagrange, Patrice Meunier, François Nadal, Christophe Eloy. Precessional instability of a fluid cylinder. Journal of Fluid Mechanics, 2011, 666, pp.104-145. 10.1017/S0022112010004040 . hal-03347572

\section{HAL Id: hal-03347572 \\ https://hal.science/hal-03347572}

Submitted on 17 Sep 2021

HAL is a multi-disciplinary open access archive for the deposit and dissemination of scientific research documents, whether they are published or not. The documents may come from teaching and research institutions in France or abroad, or from public or private research centers.
L'archive ouverte pluridisciplinaire HAL, est destinée au dépôt et à la diffusion de documents scientifiques de niveau recherche, publiés ou non, émanant des établissements d'enseignement et de recherche français ou étrangers, des laboratoires publics ou privés. 


\title{
Precessional instability of a fluid cylinder
}

\author{
By ROMAIN L GRA NGE ${ }^{1}$, PATRICE MEU NIER , \\ FRA NCSOIS NADAL AND CHRISTOPHE EL OY \\ ${ }^{1}$ IRPHE, CNRS, Aix-Marseille Université, 49 rue Joliot-Curie, 13013 Marseille, France \\ ${ }^{2}$ Commissariat à l'Energie Atomique, CESTA, 33114 le Barp, France
}

(Received 25 mai 2010)

In this paper, the instability of a fluid inside a precessing cylinder is addressed theoretically and experimentally. The precessional motion forces Kelvin modes in the cylinder which can become resonant for given precessional frequencies and cylinder aspect ratios. When the Reynolds number is large enough, these forced resonant Kelvin modes eventually become unstable. A linear stability analysis based on a triadic resonance between a forced Kelvin mode and two additional free Kelvin modes is carried out. This analysis allows to predict the spatial structure of the instability and its threshold. These predictions are compared to the vorticity field measured by Particle Image Velocimetry with an excellent agreement. When the Reynolds number is further increased, nonlinear effects appear. A weakly nonlinear theory is developed semi-empirically by introducing a geostrophic mode which is triggered by the nonlinear interaction of a free Kelvin mode with itself in the presence of viscosity. Amplitude equations are obtained coupling the forced Kelvin mode, the two free Kelvin modes and the geostrophic mode. They show that the instability saturates to a fixed point just above threshold. Increasing the Reynolds number leads to a transition from a steady saturated regime to an intermittent flow in good agreement with experiments. Surprisingly, this weakly nonlinear model still gives a correct estimate of the mean flow inside the cylinder even far from the threshold when the flow is turbulent.

\section{Introduction}

The goal of this paper is to explain the onset of turbulence observed in a precessing cylinder completely filled by a fluid.

The motion of precession is obtained when an object is in rotation around an axis which is itself rotating around a second axis. The knowledge of the flow forced by a precessional motion is of critical importance in several domains. In aeronautics (see Stewartson 1958; Gans 1984; Garg et al. 1986; Agrawal 1993; Bao \& Pascal 1997; Lambelin et al. 2009), the presence of a fluid (such as a propellant liquid) inside a flying object (spacecraft, rocket, satellite...) can have dangerous consequences on the stability of this object. Indeed the presence of a weak precessing angle, due to a non-axisymmetry of the object, can lead to a large amplitude of the contained flow when it is resonant. This flow can in turn create a torque on the flying object which destabilizes the precessing angle and thus lead to the deviation of its trajectory. A good understanding of the behavior of such a fluid-structure coupled system requires a precise knowledge of the hydrodynamics of the contained fluid.

In external geophysics, atmospheric vortices such as hurricanes or tornadoes are also subject to precessional forcing. Indeed, their axes rotate with the earth rotation around the polar axis. The fluid is thus in precession with an angle $\alpha$ equal to the co-latitude. 
This is exactly the kind of flow which is studied in this paper. Usually, the precession of atmospheric vortices is neglected and only Coriolis effects are taken into account. However, the question remains whether precession has a significative effect on the cyclone dynamics and notably on its stability.

This problem is also relevant to internal geophysics. Indeed, the Earth precession induces a forcing on its liquid core and thus generates a strong turbulent flow. Because the Earth core is made of a conductive fluid (melt iron) such a flow could be responsible for the geodynamo effect which generates the Earth magnetic field, (see Malkus 1968; Kerswell 1996; Tilgner 2005, 2007; Wu \& Roberts 2008, 2009). However, other effects such as convection, boundary layers, elliptic or tidal instability have also been proposed to explain the source of energy for the geodynamo.

The dynamics of a fluid inside a precessing spheroidal cavity was first studied by Poincaré (1910). He showed that the flow is a solid body rotation around an axis of rotation which is undetermined in the absence of viscosity. By introducing the viscosity in a thin boundary layer (Ekman layer), Busse (1968) determined this axis of rotation and the amplitude of the rotation. He also determined the critical latitudes where occurs a breakdown of the Ekman layer. This breakdown gives rise to strong shear zones in the bulk of the flow. These shear zones can be axisymmetric and are thus very well visualized in experiments (see Malkus 1968; Vanyo et al. 1995; Noir 2000). Noir et al. (2003) numerically showed that they are due to nonlinear interactions in the Ekman layer. This breakdown of the flow also excites inertial waves which propagate along characteristic surfaces at a specific angle. They were predicted asymptotically by Kerswell (1995), studied numerically by Hollerbach \& Kerswell (1995); Tilgner (1999a,b); Noir et al. (2001b) and observed experimentally by Noir et al. (2001a). Finally, when the precession rate is sufficiently large, Malkus (1968) and Vanyo et al. (1995) observed that the flow is unstable and rapidly degenerates into a turbulent state. However, it is still unclear whether this turbulent flow is due to a local instability in the Ekman layer or in the shear zones or if it results from a global instability such as a triadic resonance (Lorenzani \& Tilgner 2001; Kerswell 1993).

The presence of inertial waves in a precessing spheroid is simply due to the presence of the Coriolis force linked to the global rotation of the fluid. In a cylindrical geometry, inertial waves are also present and interfere in order to construct global modes of the fluid flow, known as Kelvin modes (Kelvin 1880). These modes are neutral in the absence of viscosity, with a frequency smaller than twice the angular velocity of the fluid (Saffman 1992). By taking into account viscous boundary layers on the walls of the cylinder Kudlick (1966) and Greenspan (1968) have extended the invscid theory to predict the viscous decay rates of a Kelvin mode. They were experimentally confirmed by McEwan (1970) and Kobine (1995) and numerically by Kerswell \& Barenghi (1995).

The precession can excite the Kelvin modes if they have the same frequency as the precession. This was first shown experimentally by McEwan (1970) who used a tilted top end rotating at a different angular velocity than the cylinder, in order to model the forcing by precession. He observed through Kalliroscope visualisations that the flow becomes resonant at the frequencies of the Kelvin modes with the correct wavelength. This was further confirmed experimentally by Manasseh (1992) and Kobine (1995) for a real precessing cylinder. The amplitude of these modes can be predicted theoretically outside of their resonances by a simple linear and inviscid theory. However, at the resonance, viscous effects have to be added in order to predict the amplitude saturation. Gans (1970b) showed that this amplitude scales as the square root of the Reynolds number when viscous effects are taken into account because of Ekman pumping inside the boundary layers. Meunier et al. (2008) have extended this result by adding the effect of nonlinear 
interactions of the Kelvin modes, which leads to the appearance of a geostrophic motion inside the cylinder.

McEwan (1970) and later Manasseh (1992) also showed that the flow is highly unstable in the vicinity of a resonant frequency. Indeed, after persisting in an apparently laminar form, they observed that the flow becomes unstable and degenerates abruptly into a fine-scale disordered state. They called this transition to turbulence the phenomenon of "resonant collapse" because it was associated with an amplitude decrease of the resonant Kelvin mode. This breakdown behavior was also observed by Thompson (1970) who studied the case of a partly filled and tilted cylinder. By performing experiments in a precessing cylinder, Manasseh (1992) catalogued different breakdown regimes by a letter scheme $(\mathrm{A}-\mathrm{G})$, where Type A breakdown results in the generation of turbulence with the smallest scales. McEwan (1970), Thompson (1970) and Manasseh (1992) also observed that for a precise range of parameters the unstable flow can relaminarize after breakdown leading to a cycle of breakdowns and relaminarizations. Because their experiments did not give any informations on global fluid velocities, the physical mechanism leading to breakdown was unclear. Mahalov (1993) showed theoretically that the precessing flow inside an infinite cylinder is unstable and that the instability mechanism is triggered by a triadic resonance between the vertical shear created by the precession force and two free Kelvin modes. However, this mechanism does not explain why the flow is more unstable at the resonance of a forced Kelvin mode. Recently, Lagrange et al. (2008) showed that the unstable flow indeed exhibits two free Kelvin modes which satisfy the conditions for a triadic resonance with the resonant forced Kelvin mode and not with the vertical shear. This mechanism will be used in this paper to carry out a linear stability analysis.

The mechanism of triadic resonance has been extensively studied to explain the elliptical instability (Moore \& Saffman 1975; Tsai \& Widnall 1976) as an interaction between the ellipticity of the vortex and two free Kelvin modes. The growth rate of this instability was later calculated analytically using local theories by Bayly (1986) and Waleffe (1990). Theoretical predictions have been widely validated numerically (Mason \& Kerswell 1999) and experimentally (Eloy et al. 2000, 2003). Such a triadic resonance occurs when the difference in the wavenumbers and frequencies between the two free Kelvin modes is equal to the wavenumber and frequency of the elliptical forcing (see the review by Kerswell 2002). Such a mechanism can be generalized to the destabilization of a Kelvin mode by the interaction of three Kelvin modes, as proposed by Mason \& Kerswell (1999) and Kerswell (1999) to explain the secondary instability of the elliptic instability. In this paper, we will use exactly the same procedure, except that the Kelvin mode is now forced by precession instead of being forced by an elliptic instability.

The paper is organized as follows. Section 2 presents the problem of a precessing cylinder and the experimental setup. Section 3 is dedicated to the base flow. The governing equations are derived and the classical linear and inviscid theory is recalled. Viscous effects are also added in order to calculate the base flow inside a resonant cylinder. A linear stability analysis based upon a mechanism of triadic resonance between Kelvin modes is developed in $\S 4$. The results (growth rate,stability diagram...) are discussed and compared with experiments in $\S 5$. Section 6 is dedicated to a weakly nonlinear theory taking into account the influence of a geostrophic mode. This theory is also compared to experimental results. Conclusions and discussion in the more general context of rotating flows are finally given in $\S 7$. 


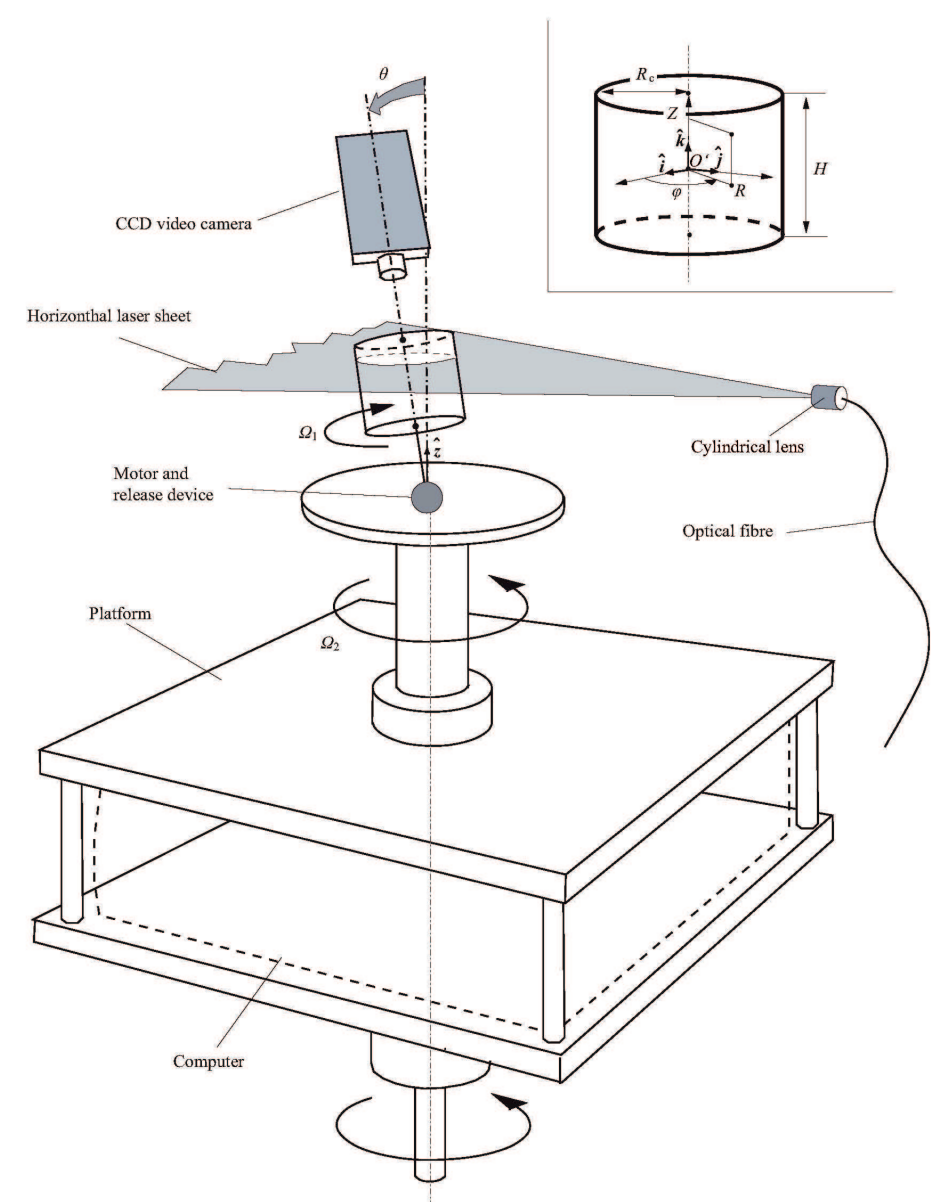

Figure 1. Sketch of the experimental setup. A cylinder of radius $R_{c}$ and height $H$ rotates around its axis at the angular velocity $\Omega_{1}$. It is mounted on a platform which rotates at the angular velocity $\Omega_{2}$. The angle between the two axes of rotation is the precessing angle $\theta$. A camera fixed above the cylinder in the platform frame is used to perform PIV measurements in the luminous plane of a horizontal laser sheet. Polar coordinates $(R, \varphi, Z)$ are defined in the cylinder rotating frame.

\section{Presentation of the problem and experimental setup}

An experimental setup has been built to study the flow inside a precessing cylinder. This experimental setup is sketched in figure 1 and described briefly in the following. Readers should refer to Meunier et al. (2008) for an extensive description.

A right cylinder of height $H$ and radius $R_{c}$ filled with water rotates at the angular velocity $\Omega_{1}$ around its axis and is mounted on a platform which rotates at the angular velocity $\Omega_{2}$. Each axis having its own driving motor, these angular velocities can be varied independently. The angular velocity $\Omega_{1}$ can be increased up to $60 \mathrm{rad} \mathrm{s}^{-1}$ and is measured with an accuracy of $0.1 \%$. The angular velocity of the platform $\Omega_{2}$ can only be varied from 0.1 to $6 \mathrm{rad} \mathrm{s}^{-1}$ and is measured with an accuracy of $0.2 \%$ when the precession frequency is larger than $0.2 \mathrm{rad} \mathrm{s}^{-1}$. Two different cylinders with the same aspect ratio $h=H / R_{c}=1.62 \pm 0.3 \%$ but different dimensions $(H=2.72 \mathrm{~cm}$ and $H=7.50 \mathrm{~cm})$ are used. Because the Reynolds number depends on $R_{c}$, these two cylinders allow to vary the Reynolds number over a large range [1000, 50000]. 
The cylinder axis is tilted relative to the axis of the platform with an angle $\theta$, which can be increased up to $15^{\circ}$ and which is determined with an absolute accuracy of $\pm 0.1^{\circ}$. A release device mounted on the platform and controlled externally allows to tilt the cylinder during the motion of the platform. The release device is composed of an electromagnet designed to keep the cylinder in a vertical position during the spin-up phase. Once the electromagnet is turned off, a drawback spring pulls the cylinder into its tilted position.

PIV measurements have been performed by seeding the flow with small reflecting polycrystalline particles (Optimage Ltd.) of mean diameter $50 \mu \mathrm{m}$ and density $1000 \pm$ $20 \mathrm{~kg} \mathrm{~m}^{-3}$. They are illuminated with a light sheet of thickness $2 \mathrm{~mm}$ created by a yttrium aluminum garner (YAG) pulsed laser. An external cylindrical lens is used to provide this laser sheet which is fixed relative to the laboratory frame. However, because the precessing angle $\theta$ is small, the laser sheet can be considered normal to the cylinder axis at first order. The height $z$ of the laser sheet can be varied along the height of the cylinder. In our experiments we have chosen $z=0$ (midheight of the cylinder) and $z=h / 4$. These two positions allow to measure a maximum transverse velocity for the Kelvin modes observed in our experiments.

The images of particles are recorded by a PIV camera (Kodak Megaplus ES 1.0, 1008× 1018 pixels) mounted on the rotating platform and aligned with the axis of the cylinder. The time interval between two successive images is relatively large (from $2 \mathrm{~ms}$ to $50 \mathrm{~ms}$ ) such that the cylinder rotates of approximately 20 degrees between the two images. This creates large displacements of the particles at the periphery of the cylinder ( 150 pixels), but the two images are rotated around the center of the cylinder in order to remove the solid body rotation of the particles. The PIV thus gives directly the velocity field in the cylinder reference frame. This procedure allows to measure very small velocities down to $1 \%$ of the velocity of the cylinder wall. Such measurements would not have been possible without the image rotation. The pairs of images are then treated by a crosscorrelation algorithm detailed in Meunier \& Leweke (2003) which gives velocity fields with $60 \times 60$ vectors. The power is brought up to the platform by a rotating collector through the vertical axis to supply the camera, the cylinder motor, the electro-magnet and the acquisition computer.

For the acquisition of a PIV field we proceed as follows. The cylinder is first kept vertical and rotates at $\Omega_{1}$. The platform rotates at $\Omega_{2}$. Once the spin-up stage is completed (i.e the flow is in solid body rotation), the cylinder is tilted in precession and a first acquisition of 85 PIV fields is launched. This first acquisition allows the study of the transient stage of the instability, i.e. the growth of the Kelvin modes. A second PIV acquisition of 85 PIV fields is started when the transient stage is completed; usually 10 minutes later. This second acquisition allows the study of the permanent stage.

Some visualizations were also performed by using Kalliroscope particles which are known to reveal the structure of the flow in a rotating fluid (see McEwan 1970; Manasseh 1992). The tank is illuminated from the side with a vertical luminous sheet created by a 5 Watts Argon laser going through a cylindrical lens. A very slight shearing motion in any section of the cylinder is sufficient to align the Kalliroscope particles, thus changing the light intensity seen by an observer. This provides an extremely sensitive indicator of relative fluid motion. This method does not give any quantitative results but is a quick and efficient way to determine the wavelength of the base flow or to see if the flow is stable or unstable. 


\section{Base flow}

In this section we give the governing equations for a precessing flow and we derive the classical solution which is valid before the appearance of the instability. This solution is linear and inviscid when the flow is non-resonant, but viscous effects have to be taken into account when one Kelvin mode is resonant. This solution will be used as a base flow for the linear stability analysis carried out in $\S 4$.

\subsection{Formulation}

A cylinder with radius $R_{c}$ and height $H$ is completely filled of a fluid with density $\rho$ and kinematic viscosity $\nu$. As shown in figure 1 , the cylinder rotates around its own axis $\left(O^{\prime}, \hat{\mathbf{k}}\right)$ at the constant angular frequency $\Omega_{1}$. The cylinder is mounted on a platform rotating at the constant angular frequency $\Omega_{2}$ around an axis $\hat{\mathbf{z}}$. There is an angle $\theta$ between the two axes of rotation creating a precessing motion of the cylinder. The rotation vector of the cylinder in the laboratory reference frame is given by $\Omega=\Omega_{1} \hat{\mathbf{k}}+\Omega_{2} \hat{\mathbf{z}}$ and is time-dependent because $\hat{\mathbf{k}}$ rotates around $\hat{\mathbf{z}}$.

To have simple boundary conditions, it is easier to solve the problem in the cylinder frame of reference $\left(O^{\prime}, \hat{\mathbf{i}}, \hat{\mathbf{j}}, \hat{\mathbf{k}}\right),\left(O^{\prime}\right.$ being the center of mass of the cylinder $)$ in which the radius vector $\mathbf{R}$ is defined by its cylindrical coordinates $(R, \varphi, Z)$ (see Fig. 1$)$. In this reference frame, the Navier-Stokes equations satisfied by the velocity field $\mathbf{U}$ and the pressure field $P$ for an incompressible fluid are

$$
\begin{gathered}
\frac{\partial \mathbf{U}}{\partial T}+(\mathbf{U} \cdot \boldsymbol{\nabla}) \mathbf{U}+2 \boldsymbol{\Omega} \times \mathbf{U}+\boldsymbol{\Omega} \times(\boldsymbol{\Omega} \times \mathbf{R})+\frac{\mathrm{d} \boldsymbol{\Omega}}{\mathrm{d} T} \times \mathbf{R}+\boldsymbol{\Gamma}_{O^{\prime}}=-\frac{1}{\rho} \boldsymbol{\nabla} P+\nu \Delta \mathbf{U}, \\
\boldsymbol{\nabla} \cdot \mathbf{U}=0,
\end{gathered}
$$

with the viscous boundary condition $\mathbf{U}=\mathbf{0}$ on the cylinder walls.

In equation (3.1a), the first two terms are the usual inertial terms, the third and fourth term are the Coriolis and centrifugal accelerations, and the fifth term is the acceleration of the rotation vector. The last left-hand side term is potential and refers to the acceleration of the centroid $O^{\prime}$ of the cylinder. The two right-hand side terms of $(3.1 a)$ are the usual pressure and viscous terms.

By using $R_{c}$ and $\Omega^{-1}=\left(\Omega_{1}+\Omega_{2} \cos \theta\right)^{-1}$ as characteristic length and time, the Navier-Stokes equations for the dimensionless velocity field $\mathbf{u}(\mathbf{r}, t)$ become

$$
\begin{aligned}
& \frac{\partial \mathbf{u}}{\partial t}+2 \hat{\mathbf{k}} \times \mathbf{u}+\nabla p=-2 \text { Ro } \omega r \cos (\omega t+\varphi) \hat{\mathbf{k}} \\
&+\mathbf{u} \times(\boldsymbol{\nabla} \times \mathbf{u})-2 \operatorname{Ro} \boldsymbol{\delta} \times \mathbf{u}+\frac{\Delta \mathbf{u}}{R e}, \\
& \boldsymbol{\nabla} \cdot \mathbf{u}=0
\end{aligned}
$$

with

$$
\omega=\frac{\Omega_{1}}{\Omega}, R o=\frac{\Omega_{2} \sin \theta}{\Omega}, R e=\frac{\Omega R_{c}^{2}}{\nu}, \delta=\cos (\omega t) \hat{\mathbf{i}}-\sin (\omega t) \hat{\mathbf{j}}
$$

In this dimensionless form, $h=H / R_{c}$ is the aspect ratio of the cylinder. The dimensionless pressure field $p(\mathbf{r}, t)$ includes all the potential terms (see Meunier et al. 2008, for the detail of $p$ ). The boundary condition of the velocity field is

$$
\mathbf{u}=\mathbf{0} \text { at the walls }(r=1 \text { or } z= \pm h / 2) .
$$

The Navier-Stokes equations $(3.2 a, b)$ with the boundary condition (3.4) govern the flow inside a precessing cylinder. This set of equations has been obtained without any 
approximation and is thus valid for any value of the experimental parameters. As it appears from these equations, the problem is entirely governed by four dimensionless parameters. The amplitude of the forcing term is called a Rossby number $R o$ because it represents the ratio between the vorticity of the flow and the solid body rotation $\Omega$. The dimensionless frequency $\omega$ of the forcing term fixes the frequency of the Kelvin modes which are excited. The cylinder aspect ratio $h$ selects the structure of the Kelvin modes, and the Reynolds number $R e$ quantifies the viscous damping. In the following we will limit ourselves to the case of asymptotically small Rossby number $R o$ and large Reynolds number Re. This assumption is coherent with atmospherical and geophysical observations. It is also the relevant limit for a stable flow or at the onset of instability.

In the following the four-component and complex vector associated to the velocitypressure field $(\mathbf{u}, p)$ will be noted $\mathbf{v}$, such that $(\mathbf{u}, p)=\mathbf{v}+\overline{\mathbf{v}}$ with $\overline{\mathbf{v}}$ the complex conjugate of $\mathbf{v}$. Using this formulation the Navier-Stokes equations $(3.2 a, b)$ rewrite

$$
\begin{aligned}
\left(\frac{\partial}{\partial t} \mathcal{I}+\mathcal{M}\right) \mathbf{v}+\text { c.c. }=R o \mathbf{F}_{0} \mathrm{e}^{\mathrm{i}(\omega t+\varphi)}+\mathbf{N}(\mathbf{v}, \mathbf{v}) & +R o\left(\mathcal{D} \mathrm{e}^{\mathrm{i}(\omega t+\varphi)}+\text { c.c. }\right) \mathbf{v} \\
& +\frac{\mathcal{L} \mathbf{v}}{R e}+\text { c.c. }
\end{aligned}
$$

where the operators $\mathcal{D}, \mathcal{I}, \mathcal{L}, \mathcal{M}$, the forcing vector $\mathbf{F}_{0}$ and the bilinear function $\mathbf{N}$ are defined in Appendix A. The symbol c.c. stands for the complex conjugate.

\subsection{Non-resonant cylinder}

In order to solve (3.5), an asymptotically small Rossby number $R o$ and a large Reynolds number $R e$ are assumed. In this limit $\mathbf{v}$ is $O(R o)$ and the Navier-Stokes equation (3.5) at order $O(R o)$ becomes

$$
\left(\frac{\partial}{\partial t} \mathcal{I}+\mathcal{M}\right) \mathbf{v}=R o \mathbf{F}_{0} \mathrm{e}^{\mathrm{i}(\omega t+\varphi)}
$$

The no-slip boundary condition (3.4) becomes at this order a condition of no outward flow

$$
\mathbf{u} \cdot \mathbf{n}=0 \text { at the walls }(r=1 \text { or } z= \pm h / 2),
$$

where $\mathbf{n}$ is an unitary vector normal to the wall.

The set of equations (3.6) and (3.7) is a linear system whose second term represents the forcing due to precession. This system admits a particular solution $\mathbf{v}_{\text {part. }}=$ $\left(0,0, \operatorname{Ro}\right.$ i $\left.r \mathrm{e}^{\mathrm{i}(\omega t+\varphi)}, 0\right)$ which is a shear along the cylinder axis. Because it does not satisfy the boundary condition (3.7) at $z= \pm h / 2$, it must be completed with a solution of the homogeneous equation (i.e. equation (3.6) without forcing term). Due to time and azimuthal dependence of the forcing and particular solution, the homogeneous solution can be written a sum of Kelvin modes of azimuthal wavenumber $m=1$ and angular frequency $\omega$ (see Greenspan 1968). Gathering the particular solution and the Kelvin modes yields the solution of (3.6) and (3.7)

$$
\mathbf{v}=\mathbf{v}_{\text {part. }}+\sum_{i=1}^{\infty} \varepsilon_{i} \mathbf{v}_{1, \omega, k_{i}},
$$

where $\mathbf{v}_{1, \omega, k_{i}}$ is a Kelvin mode of azimuthal wavenumber $m=1$, frequency $\omega$ and axial wavenumber $k_{i}$. Its structure is composed of two travelling waves of opposite wavenumber $\pm k_{i}$ in order to form a stationary wave and match the parity of the forcing

$$
\mathbf{v}_{1, \omega, k_{i}}=\mathbf{u}_{1, \omega, k_{i}}(r) \mathrm{e}^{\mathrm{i}\left(\omega t+\varphi+k_{i} z\right)}-\mathbf{u}_{1, \omega,-k_{i}}(r) \mathrm{e}^{\mathrm{i}\left(\omega t+\varphi-k_{i} z\right)} .
$$


The vector $\mathbf{u}_{m, \omega, k_{i}}$ is found by solving the homogeneous equation and is given in Appendix A. The boundary condition in $r=1$ imposes that the axial wavenumber $k_{i}$ is the positive root of the constitutive relation

$$
\delta_{i}^{2}=\frac{4-\omega_{i}^{2}}{\omega_{i}^{2}} k_{i}^{2}
$$

where the radial wavenumber $\delta_{i}$ is solution of Kelvin's dispersion relation $D\left(1, \omega, \delta_{i}\right)=0$ with

$$
D(m, \omega, \delta)=\omega \delta J_{m}^{\prime}(\delta r)+2 m J_{m}(\delta r) .
$$

This condition ensures that $\mathbf{v}$ vanishes at $r=1$. In equation (3.11) $J_{m}(x)$ is the Bessel function of the first kind and $J_{m}^{\prime}(x)$ its $x$-derivative. For a given azimuthal wavenumber and for a given frequency $\omega$ such that $-2<\omega<2$, the dispersion relation (3.11) admits an infinite and countable number of real roots $\delta_{i}$ numbered in ascending order. The $i$-th roots corresponds to the $i$-th Kelvin mode.

A PIV measurement of the first Kelvin mode $(i=1)$ is shown in figure $2(a)$. It represents the axial and instantaneous vorticity field $\zeta$. This mode is characterized by two counter rotating vortices which induce a velocity along the $y$-axis (tilted axis). In the general case, the $i$-th Kelvin mode contains $2 i$ vortices.

The amplitude $\varepsilon_{i}$ of each Kelvin mode is real and can be calculated by imposing a vanishing velocity at $z= \pm h / 2$. For this purpose, the $z$-velocity of the particular solution $\mathbf{v}_{\text {part. }}$ is decomposed on the set of Bessel functions which characterize the $z$-component of the Kelvin modes. It leads to the inviscid and nonlinear amplitude $\varepsilon_{i}=R o a_{i}$ of the Kelvin modes, where $a_{i}$ is given in (A 8) of Appendix A. The linear forced response as a function of the forcing frequency $\omega$ is plotted in figure $2(b)$ for the first Kelvin mode. It shows a series of divergences corresponding to the natural frequencies $\omega_{i, n}$ of the cylinder (the notation $\omega_{i, n}$ stands for the $n$-th resonance of the $i$-th Kelvin mode). These resonances occur when the $z$-velocity of the Kelvin mode vanishes, i.e. when the wavenumber $k_{i}$ of the Kelvin mode is equal to $(2 n-1) \pi / h$ with $n$ the number of the resonance.

It can be demonstrated (Kudlick 1966) that there is always a Kelvin mode arbitrarily close to a resonance for any forcing frequency $\omega$. It is thus necessary to add viscous effects in order to predict the saturation of the Kelvin mode amplitude $\varepsilon_{i}$ at a resonance. These results are recalled in the next section.

\subsection{Resonant cylinder}

To predict the saturation of the amplitude at a resonant frequency it is necessary to include the viscous effects (Gans 1970b) and/or the nonlinear effects (Meunier et al. 2008). In the present paper, the amplitude is always saturated by viscous effects because $\left|R e^{1 / 2} R o^{2 / 3}\right| \ll 1$. In other words, the instability appears before nonlinearities become significant in the base flow. Moreover, because volume viscous effects are $O\left(\left(\mathrm{~m}^{2}+\right.\right.$ $\left.\left.\delta_{i}^{2}\right) R e^{-1}\right)=O\left(R e^{-1}\right)$ and surface viscous effects are $O\left(R e^{-1 / 2}\right)$ volume viscous effects will be neglected for the saturation of the resonant Kelvin mode amplitude. As shown by Gans (1970b), the resonant Kelvin mode of amplitude $\varepsilon_{i}$ generates a secondary flow at order $O\left(\varepsilon_{i} R e^{-1 / 2}\right)$ in the core of the cylinder due to Ekman pumping in the viscous boundary layers. The correct scaling is obtained when this secondary flow is of the order of the forcing amplitude $R o$. This gives a mode amplitude $\varepsilon_{i}=O\left(R o R e^{1 / 2}\right)$ which is $R e^{1 / 2}$ larger than the flow in the non-resonant case. Applying a solvability condition on the secondary flow allows to calculate analytically the amplitude of the resonant Kelvin 


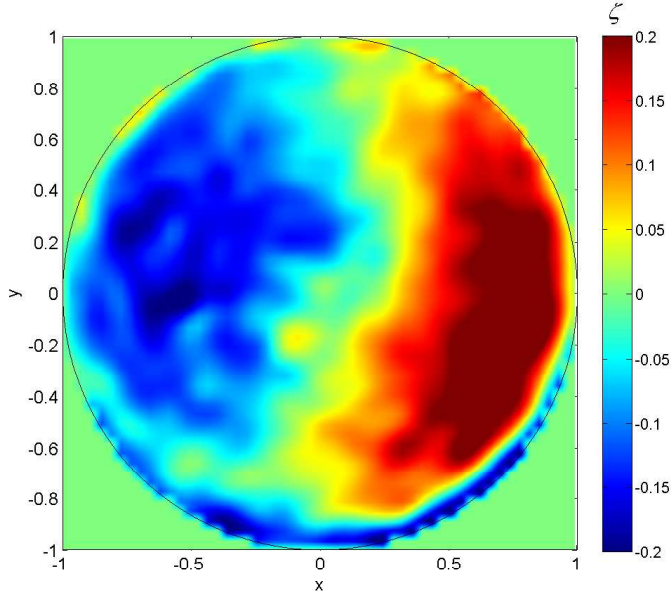

(a)

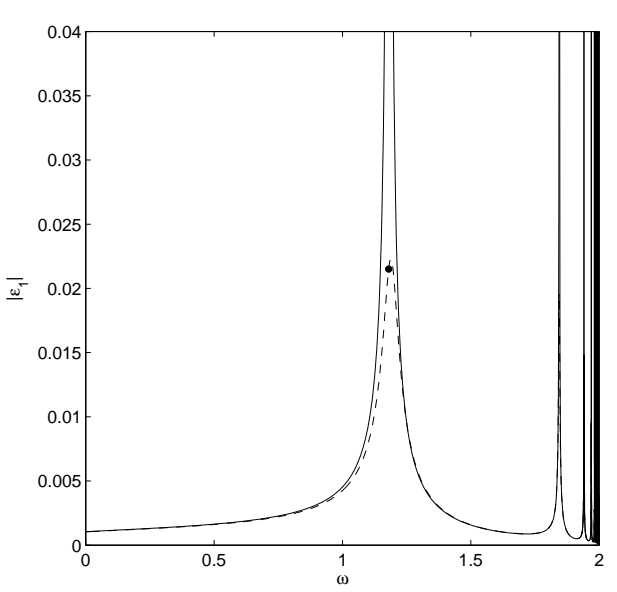

(b)

Figure 2. (a) Axial vorticity field $\zeta$ of the first Kelvin mode measured by PIV at its first resonance $(\omega=1.18)$, in the absence of instability. (b) Amplitude of the first Kelvin mode as a function of $\omega$. The solid line corresponds to the linear and inviscid theory given by equation (A 8) and the dashed line to the viscous theory given by equation (3.12). The point is the value of $\left|\varepsilon_{1}\right|$ for $\omega=1.18$ at the exact resonance. $h=1.62, R e \approx 3500$ and $R o=-0.0031$.

mode close, and at the resonance (Meunier et al. 2008) :

$$
\varepsilon_{i}=\frac{\mathrm{i} f R o}{\frac{s}{R e^{1 / 2}}+\mathrm{i} \frac{f}{a_{i}}},
$$

where the linear forcing parameter $f$ is real and given in Appendix A. The surface viscous parameter $s$ is a complex number with a positive real part and is given in Appendix B. The term $1 / a_{i}$ represents the detuning damping of the forced Kelvin mode if the frequency ratio $\omega$ is not exactly equal to a resonance frequency. This viscous amplitude is represented as a dashed line on figure $2(b)$ for $R e=3500$. It is close to the inviscid amplitude $\varepsilon_{i}=R o a_{i}$ (solid line) far from the resonance and saturates at the resonance (i.e. when $a_{i}=\infty$ ) at a finite value $\varepsilon_{i}=f R o R e^{1 / 2} / s$ represented by a point. It can be noted that the mode amplitude is maximum when the detuning of the forcing frequency compensates exactly the viscous detuning, i.e. $f / a_{i}=-R e^{-1 / 2} \operatorname{Im}(s)$ and not at the exact resonance.

At resonance, the base flow is an order $R e^{1 / 2}$ larger than far from the resonance and may thus be subject to stronger instabilities. In the following, the forcing frequency $\omega$ will be assumed to be a natural frequency of the cylinder such that the base flow is composed of a predominant Kelvin mode $\mathbf{v}_{1, \omega, k_{i}}$ with an amplitude $\varepsilon_{i}$ given by (3.12). For the sake of clarity the index $i$ will be dropped such that the amplitude of the resonant Kelvin mode is noted $\varepsilon$ instead of $\varepsilon_{i}$. Its axial wavenumber is simply noted $k$ and its radial wavenumber $\delta$. We also assume that $\left|R o R e^{1 / 2}\right| \ll 1$ such that $\varepsilon$ is a small parameter. This assumption allows to carry out an asymptotic and linear stability analysis of the base flow described here.

\section{Linear stability analysis}

As described in the literature (see Manasseh 1996; Kobine 1996), when the Reynolds number or the Rossby number is increased above a certain threshold (which depends on $h$ 
and $\omega$ ), the flow inside the cylinder becomes unstable. PIV measurements in two different sections of the cylinder have revealed the three-dimensional structure of this instability (see Lagrange et al. 2008). It is composed of two free Kelvin modes whose wavenumbers and frequencies respect the conditions for a triadic resonance with the forced Kelvin mode, in a similar manner as for the elliptical instability (see Malkus 1989; Eloy et al. 2000; Kerswell 2002). In this paper, a linear stability analysis based on such a mechanism is presented. It allows to predict the structure of the instability, its growth rate, and the stability threshold.

\subsection{Governing equations for the perturbation}

To perform a stability analysis, a perturbation defined by its four component and complex velocity-pressure field $\widetilde{\mathbf{v}}$ is added to the base flow. The total flow $\mathbf{v}$ in the precessing cylinder is thus the sum of the resonant base flow $\mathbf{v}_{1, \omega, k}$ (given by (3.9)), whose amplitude $\varepsilon$ is given by (3.12), the perturbation $\widetilde{\mathbf{v}}$ and some lower order terms : the particular solution $\mathbf{v}_{\text {part. }}$ and the non-resonant Kelvin modes $\mathbf{v}_{1, \omega, k_{i}}$

$$
\mathbf{v}=\varepsilon \mathbf{v}_{1, \omega, k}+\widetilde{\mathbf{v}}+\mathbf{v}_{\text {part. }}+\sum_{i} \varepsilon_{i} \mathbf{v}_{1, \omega, k_{i}}
$$

We recall that the dominant term $\varepsilon \mathbf{v}_{1, \omega, k}$ is of order $R o R e^{1 / 2}$. The other terms of the base flow ( $\mathbf{v}_{\text {part. }}$ and $\sum \varepsilon_{i} \mathbf{v}_{1, \omega, k_{i}}$ ) are of order $R o$.

Substituting this decomposition (4.1) into (3.5) yields the equation satisfied by the perturbation $\widetilde{\mathbf{v}}$ :

$$
\left(\frac{\partial}{\partial t} \mathcal{I}+\mathcal{M}\right) \widetilde{\mathbf{v}}+\text { c.c. }=\mathbf{N}\left(\widetilde{\mathbf{v}}, \varepsilon \mathbf{v}_{1, \omega, k}\right)+\mathbf{N}\left(\varepsilon \mathbf{v}_{1, \omega, k}, \widetilde{\mathbf{v}}\right)+\frac{1}{R e} \mathcal{L} \widetilde{\mathbf{v}}+\text { o.t. }+ \text { c.c. }
$$

We recall that $\mathcal{I}, \mathcal{M}, \mathbf{N}$ and $\mathcal{L}$ are operators given in Appendix A. The left-hand side term of equation (4.2) is the linear unsteady term of the homogeneous equation. The first and second right-hand side terms represent the nonlinear interaction between the resonant Kelvin mode and the perturbation. They are of order $O\left(R o R e^{1 / 2} \widetilde{\mathbf{v}}\right)$. The third term is the classical viscous term for the perturbation. It is of order $O\left(R e^{-1} \widetilde{\mathbf{v}}\right)$. The notation o.t. stands for 'other terms' and includes the nonlinear interaction of the four-component vector $\mathbf{v}_{\text {part. }}+\sum \varepsilon_{i} \mathbf{v}_{1, \omega, k_{i}}+\widetilde{\mathbf{v}}$ with itself and the term $R o\left(\mathcal{D} \mathrm{e}^{\mathrm{i}(\omega t+\varphi)}+\right.$ c.c. $) \mathbf{v}$. In these terms, the leading order is $O\left(R_{0} \widetilde{\mathbf{v}}\right)$, which is negligible compared to the nonlinear terms of order $O\left(R o R e^{1 / 2} \widetilde{\mathbf{v}}\right)$ explicited in (4.2). Terms of order $O\left(R e R o^{2}\right)$ and $O\left(\widetilde{\mathbf{v}}^{2}\right)$ are also neglected in this linear analysis. Note that the forcing term $R o \mathbf{F}_{0} \mathrm{e}^{\mathrm{i}(\omega t+\varphi)}$ from (3.5) does not appear in equation (4.2) because it has been cancelled out by the unsteady term of the particular solution.

The flow is assumed to satisfy a no-slip boundary condition

$$
\widetilde{\mathbf{v}}=\mathbf{0} \text { at the walls }(r=1 \text { or } z= \pm h / 2) \text {. }
$$

The equation (4.2) and the boundary condition (4.3) describe the evolution of the linear perturbation $\widetilde{\mathbf{v}}$ in the limit of small amplitudes $\varepsilon$.

In order to solve the system of equations (4.2-4.3) the four-component velocity-pressure field $\widetilde{\mathbf{v}}$ is expanded in powers of $\varepsilon$ as follows

$$
\widetilde{\mathbf{v}}=\mathbf{v}^{(0)}+\varepsilon \mathbf{v}^{(1)}+O\left(\varepsilon^{2}\right) .
$$

The equation for the growth rate of the instability is obtained by substituting the above expansion (4.4) into the Navier-Stokes equation (4.2) and examining its two first orders. 
Note that in this study we will consider the critical scaling $R e^{-1 / 2}=O(\varepsilon)$ such that boundary viscous effects are of the same order as $\mathbf{N}\left(\widetilde{\mathbf{v}}, \varepsilon \mathbf{v}_{1, \omega, k}\right)$ and $\mathbf{N}\left(\varepsilon \mathbf{v}_{1, \omega, k}, \widetilde{\mathbf{v}}\right)$.

\subsection{Description of the free Kelvin modes}

At order 0 , equation (4.2) is the homogeneous equation

$$
\left(\frac{\partial}{\partial t} \mathcal{I}+\mathcal{M}\right) \mathbf{v}^{(0)}=0
$$

Because viscous effects are of order $R e^{-1 / 2}$ (boundary viscous effects) or $R e^{-1}$ (volume viscous effects), an inviscid boundary condition is assumed at this order

$$
\mathbf{v}^{(0)} \cdot \mathbf{n}=0 \text { at the walls }(r=1 \text { or } z= \pm h / 2) .
$$

The resolution of the set of equations (4.5-4.6) is classical and its solution is a linear combination of free Kelvin modes. Each free Kelvin mode $\mathbf{v}_{j}$ is composed of two travelling waves of opposite wavenumber $k_{j}$ in order to satisfy the boundary condition (4.6) at the top and bottom of the cylinder

$$
\mathbf{v}_{j}=\mathbf{u}_{m_{j}, \omega_{j}, k_{j}}(r) \mathrm{e}^{\mathrm{i}\left(\omega_{j} t+m_{j} \varphi+k_{j} z\right)} \pm \mathbf{u}_{m_{j}, \omega_{j},-k_{j}}(r) \mathrm{e}^{\mathrm{i}\left(\omega_{j} t+m_{j} \varphi-k_{j} z\right)} .
$$

We recall that the expression of the four-component vector $\mathbf{u}_{m_{j}, \omega_{j}, k_{j}}$ is given in Appendix A. At this order the amplitude $A_{j}$ of a free Kelvin mode is undefined but its azimuthal wavenumber $m_{j}$, its frequency $\omega_{j}$ and its axial wavenumber $k_{j}$ are connected through the dispersion relation $D\left(m_{j}, \omega_{j}, \delta_{j}\right)=0$ given by equation (3.11) such that the radial velocity vanishes at $r=1$. In figure 3 the first branches of the dispersion relation are plotted for $m_{j}=6$ (solid line).

The condition of no normal flow at the top and the bottom discretizes the vertical wavenumber $k_{j}$ as a multiple of $\pi / h$. It also separates the free Kelvin modes into two categories with different parity, depending on the choice of sign between the two waves of opposite wavenumber $k_{j}$ in (4.7). The plus sign allows to consider free Kelvin modes whose axial velocity (resp. vorticity) is a sine (resp. cosine) function of $z$ and the axial wavenumber satisfies $k_{j}=(2 n-1) \pi / h$ with $n$ an integer. The minus sign allows to consider free Kelvin modes whose axial velocity (resp. vorticity) is a cosine (resp. sine) function of $z$, with $k_{j}=2 n \pi / h$.

To examine the mechanism of triadic resonance, the perturbation $\mathbf{v}^{(0)}$ will be assumed to be, at leading order, a combination of two free Kelvin modes $\mathbf{v}_{1}$ and $\mathbf{v}_{2}$ with unknown amplitudes $A_{1}$ and $A_{2}$

$$
\mathbf{v}^{(0)}=A_{1} \mathbf{v}_{1}+A_{2} \mathbf{v}_{2},
$$

where the amplitudes are varying slowly with time such that time derivatives will appear at next order.

\subsection{Influence of the Ekman layers}

It can be noted that the dispersion relation and the determination of the structure of the free Kelvin modes are obtained with an inviscid boundary condition on the cylinder walls (equation (4.6)). This condition does not match the viscous boundary condition given by equation (4.3). There will thus remain a wall parallel flow at the leading order for the free Kelvin modes. This problem can be solved by adding a viscous flow, in a viscous boundary layer of thickness $O\left(R e^{-1 / 2}\right)$ near the walls. However this resulting viscous flow has a component perpendicular to the walls, of order $O\left(R e^{-1 / 2}\right)$ called Ekman pumping. This pumping is an exponential decreasing function inside the Ekman 
layers. Note that in our problem we have an Ekman pumping due to the lateral wall and the top and bottom walls of the cylinder. The calculation of Ekman layers is classical. An exhaustive description is given in Greenspan (1968). For the particular case of Kelvin modes, the reader could refer to Kudlick (1966).

\subsection{Triadic resonance}

Experiments have demonstrated (see Lagrange et al. 2008) that the instability of a fluid inside a precessing cylinder is due to a triadic resonance between the resonant Kelvin mode $\varepsilon \mathbf{v}_{1, \omega, k}$ and two free Kelvin modes $\mathbf{v}_{1}$ and $\mathbf{v}_{2}$. These three modes can be coupled by the nonlinear terms $\mathbf{N}\left(\widetilde{\mathbf{v}}, \varepsilon \mathbf{v}_{1, \omega, k}\right)$ and $\mathbf{N}\left(\varepsilon \mathbf{v}_{1, \omega, k}, \widetilde{\mathbf{v}}\right)$ in equation (4.2) when their Fourier components are chosen appropriately. The nonlinear interaction between the first mode $\mathbf{v}_{1}$ and the forced mode $\mathbf{v}_{1, \omega, k}$ has a Fourier component $\mathrm{e}^{\mathrm{i}\left(\omega_{1} t+m_{1} \varphi \pm k_{1} z\right)} \mathrm{e}^{\mathrm{i}(\omega t+\varphi \pm k z)}$. This term has a Fourier component $\mathrm{e}^{\mathrm{i}\left(\omega_{2} t+m_{2} \varphi \pm k_{2} z\right)}$ identical to that of the second mode $\mathbf{v}_{2}$ only when the frequencies and wavenumbers satisfy the resonance condition

$$
\begin{gathered}
m_{2}-m_{1}=1, \\
\omega_{2}-\omega_{1}=\omega, \\
\left|k_{2}-k_{1}\right|=k .
\end{gathered}
$$

If these conditions are satisfied, the nonlinear interaction between $\mathbf{v}_{2}$ and the forced mode $\mathbf{v}_{1, \omega, k}$ has also the same Fourier component as $\mathbf{v}_{1}$. It can be noted that the last equation of this system offers two possibilities which are $k_{2}-k_{1}=k$ and $k_{1}-k_{2}=k$. However, in all our calculations the first case has always been the most unstable one.

The first condition imposes that the two free Kelvin modes have azimuthal wavenumbers separated by one. This is different from the elliptic instability where they should be separated by 2 (Kerswell 2002). This comes from the fact that, here, the forcing term has an azimuthal wavenumber $m=1$, unlike the ellipticity which has an azimuthal wavenumber $m=2$.

To find Kelvin modes satisfying the resonance condition, we plot on the same graph the dispersion relation of the second mode $\mathbf{v}_{2}$ and the dispersion relation of the first mode $\mathbf{v}_{1}$ translated by $k$ along the abscissae and by $\omega$ along the ordinate. An example is shown in Fig. 3 for $m_{2}=6$ and $m_{1}=5$. At each crossing point between the dispersion relation of mode 1 (dashed lines) and mode 2 (solid line) the conditions of resonance are fulfilled. Since there is an infinite number of branches for each mode and an infinity of possible azimuthal wavenumber $m_{2}$, there is a triple-infinite number of resonances. We will label these resonant combinations $\left(m_{2}, l_{1}, l_{2}\right)$, where $l_{1}$ (resp. $\left.l_{2}\right)$ is the branch number of the dispersion relation for the first mode (resp. second mode). As an example, the lowest order resonant combination $\left(m_{2}=6, l_{1}=1, l_{2}=1\right)$ is marked by a circle on Fig. 3 . This combination will be found to be the most unstable and it will be extensively studied and compared to the experiments. It can be noted that the study can be restricted to free Kelvin modes with $m_{j} \geq 0$ because the dispersion relation (3.11) satisfies the following symmetry $D\left(m_{j}, \omega_{j}, \delta_{j}\right)=-D\left(-m_{j},-\omega_{j}, \delta_{j}\right)$.

An additional condition arises because the free Kelvin modes must have a zero normal velocity at the top and the bottom, which quantifies the wavenumbers $k_{j}$ as multiples of $\pi / h$. It is the case in Fig. 3 where the resonant combination $(6,1,1)$ is located on a dashdotted line $\left(k_{2}=2 \pi / h\right)$. This case of exact resonance only appears for specific aspect ratios (here $h=1.62$ ) which allow an intersection between the three lines. The principal aspect ratios $h$ allowing exact resonances are listed in Table 1 (resp. Table 2 ) for the combinations $\left(m_{2}, 1,1\right)$ (resp. $\left.\left(m_{2}, 2,2\right)\right)$ when the first Kelvin mode is excited at its first 


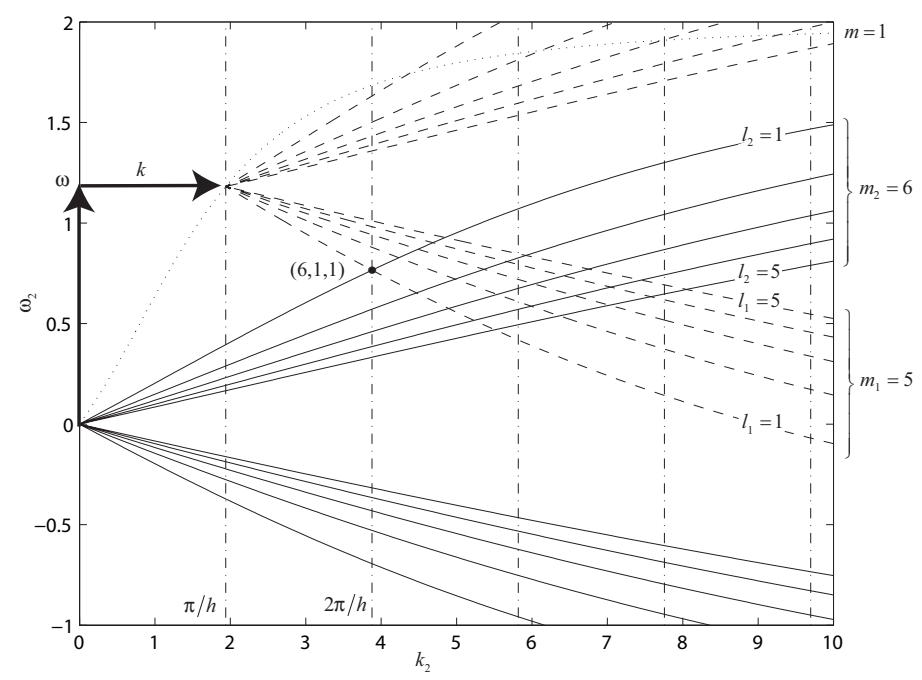

Figure 3. Dispersion relations of the free Kelvin modes $(h=1.62, \omega=1.18$ ). Solid lines (resp. dashed lines) correspond to the first five branches of the dispersion relation of the free Kelvin modes with an azimuthal wavenumber $m_{2}=6$ (resp. $m_{1}=5$ ). The dotted line represents the dispersion relation of the forced Kelvin mode $(m=1)$, which is used to translate the dispersion relations of the first mode horizontally by $k=\pi / h$ and vertically by $\omega=1.18$. Resonant free Kelvin modes correspond to a crossing point for the case $k_{2}-k_{1}=k$. Vertical dash-dotted lines indicate the discretisation of the axial wavenumber $k_{2}=n \pi / h$ for which the free Kelvin mode 'fits' inside the cylinder.

resonance and where $k_{1}=\pi / h$. Most of the resonances are obtained for small aspect ratios (for which the resonance frequency is large) and large azimuthal wavenumbers. Note that there does not exist any exactly resonant combinations $\left(m_{2}, 1,1\right)$ (resp. $\left.\left(m_{2}, 2,2\right)\right)$ with $m_{2} \leq 5$ (resp. $m_{2} \leq 3$ ). This is why the instabilities observed in the experiments usually have high azimuthal wavenumbers. Finally, it can be noted that since the forced Kelvin mode is resonant, its axial wavenumber $k$ is an odd multiple of $\pi / h$. Thus equation $(4.9 c)$ implies that the two free Kelvin modes have different parities with respect to $z$ (i.e. free Kelvin modes with a different sign in equation (4.7)). By contrast, at the anti-resonance of the forced Kelvin mode, the axial wavenumber $k$ is an even multiple of $\pi / h$. Thus the two free Kelvin modes have the same parities with respect to $z$. In this condition it can be shown that a mechanism of triadic resonance between Kelvin modes can not lead to the instability.

Most of the time a combination $\left(m_{2}, l_{1}, l_{2}\right)$ is not exactly resonant (see Manasseh 1996) because the crossing point is not on a dash-dotted line (i.e. $k_{j} \neq n \pi / h$, with $n$ an integer). The free Kelvin modes thus do not satisfy the boundary condition (4.6) at the top and bottom of the cylinder and should not be considered. However, the problem can still be solved by introducing small detuning parameters $\Delta k_{1}$ and $\Delta k_{2}$ which measure the distance between the wavenumber $k_{1}$ (or $k_{2}$ ) and the closest $n \pi / h$ :

$$
\Delta k_{j}=\min _{n \in \mathbb{N}^{*}}\left(\left|n \frac{\pi}{h}-k_{j}\right|\right) \operatorname{sgn}\left(n \frac{\pi}{h}-k_{j}\right) .
$$

In figure 3 these detunings correspond to the relative distance between a crossing point and the closer vertical dash-dotted line. These detunings will only modify the problem at order $\varepsilon$ if they are of order $O(\varepsilon)$. Note that, as long as $\omega$ is a resonant frequency, the detuning parameters satisfy $\Delta k_{1}=\Delta k_{2}$. 


$\begin{array}{ccccccc}h & 10.8 & 3.60 & 1.62 & 1.09 & 0.886 & 0.758 \\ \omega_{1,1} & 0.1639 & 0.5501 & 1.181 & 1.503 & 1.636 & 1.718 \\ m_{2} & 7 & 6 & 6 & 7 & 8 & 9 \\ n_{1} n_{2} & 1.16 & 1.07 & 4.11 & 15.4 & 34.4 & 64.5 \\ |R o R e|_{\text {crit }} & 5.41 & 7.14 & 7.11 & 8.60 & 10.4 & 12.4 \\ & & & & & & \\ h & 0.669 & 0.601 & 0.547 & 0.504 & 0.467 & 0.436 \\ \omega_{1,1} & 1.772 & 1.811 & 1.841 & 1.863 & 1.881 & 1.895 \\ m_{2} & 10 & 11 & 12 & 13 & 14 & 15 \\ n_{1} n_{2} & 108 & 170 & 253 & 358 & 496 & 666 \\ |R o R e|_{\text {crit }} & 14.5 & 16.8 & 19.2 & 21.7 & 24.3 & 27.1\end{array}$

TABLE 1. Theoretical results considering the $\left(m_{2}, 1,1\right)$ combinations with $k_{2}=2 \pi / h$ and $k_{1}=k=\pi / h$. The combination is exactly resonant only for a specific aspect ratio $h$ (given in the first row) at its associated frequency $\omega_{1,1}$ (given in the second row) and for an azimuthal wavenumber of the second mode $m_{2}$ (given in the third row). The fourth row gives the value of $n_{1} n_{2}$ which is the square of the inviscid growth rate. The last row gives the stability threshold without volume viscous effects and is calculated with equation (4.21).

\begin{tabular}{|c|c|c|c|c|c|c|c|}
\hline$h$ & \multicolumn{2}{|c|}{9.57} & 1.13 & 0.880 & 0.744 & 0.651 & 0.581 \\
\hline$\omega_{1,1}$ & \multicolumn{2}{|c|}{0.1867} & 1.477 & 1.640 & 1.727 & 1.783 & 1.822 \\
\hline$m_{2}$ & \multicolumn{2}{|c|}{4} & 4 & 5 & 6 & 7 & 8 \\
\hline$n_{1} n_{2}$ & \multicolumn{2}{|c|}{$3.15 \times 10^{-3}$} & 8.42 & 30.4 & 71.0 & 138 & 242 \\
\hline$|R o R e|_{\text {crit }}$ & \multicolumn{2}{|c|}{106} & 10.5 & 11.2 & 12.5 & 14.0 & 15.8 \\
\hline$h$ & 0.527 & 0.484 & 0.448 & 0.417 & 0.391 & 0.368 & 0.348 \\
\hline$\omega_{1,1}$ & 1.851 & 1.873 & 1.890 & 1.904 & 1.915 & 1.924 & 1.932 \\
\hline$m_{2}$ & 9 & 10 & 11 & 12 & 13 & 14 & 15 \\
\hline$n_{1} n_{2}$ & 24.3 & 36.6 & 52.9 & 74.4 & 101 & 134 & 174 \\
\hline$|R o R e|_{\text {crit }}$ & 17.6 & 19.6 & 21.7 & 23.9 & 26.2 & 28.6 & 31.0 \\
\hline
\end{tabular}

TABLE 2. Theoretical results considering the $\left(m_{2}, 2,2\right)$ combinations with $k_{2}=2 \pi / h$ and $k_{1}=k=\pi / h$.

\subsection{Amplitude Equations}

As mentioned previously, at leading order the perturbation $\widetilde{\mathbf{v}}$ is a combination of two free Kelvin modes with unknown amplitudes. We now assume that $A_{1}$ and $A_{2}$ vary slowly with time such that $\partial A_{j} / \partial t$ is of order $\varepsilon$. We will consider that this combination is not exactly resonant and that detuning effects $\Delta k_{j}$ are also of order $\varepsilon$. This 'critical scaling' allows to extend the theory in the presence of detuning effects (with this scaling detuning terms are of same order as destabilising terms). For larger detuning effects, the flow is no longer unstable.

At order $\varepsilon$ equation (4.2) becomes

$$
\begin{aligned}
\varepsilon\left(\frac{\partial}{\partial t} \mathcal{I}+\mathcal{M}\right) \mathbf{v}^{(1)}+\text { c.c. } & =\left(-\frac{\partial}{\partial t} \mathcal{I}+\mathrm{i} \Delta k_{j} \mathcal{M}_{\Delta}\right) \mathbf{v}^{(0)}+\mathbf{N}\left(\mathbf{v}^{(0)}, \varepsilon \mathbf{v}_{1, \omega, k}\right) \\
& +\mathbf{N}\left(\varepsilon \mathbf{v}_{1, \omega, k}, \mathbf{v}^{(0)}\right)+\frac{\mathcal{L} \mathbf{v}^{(0)}}{R e}+\text { c.c. }
\end{aligned}
$$

In equation (4.11) the left-hand side term is the linear operator applied to the solution at order $\varepsilon$. The first right-hand side term is composed by the slow unsteady part of 
the perturbation of order 0 and by its detuning term. The operator $\mathcal{M}_{\Delta}$ is defined in Appendix B and is obtained via a Taylor expansion of the Fourier component $\mathrm{e}^{\mathrm{i} \Delta k_{j} z}$ of the free Kelvin modes. The second and third right hand side term represent the nonlinear interaction between the resonant Kelvin mode and the two free Kelvin modes. These terms represent the mechanism of triadic resonance. The last term of this equation is the volume viscous term. It should not appear at this order but at order $\varepsilon^{2}$ since $\varepsilon=O\left(R e^{-1 / 2}\right)$. However we have decided to take it into account in the analysis because its importance in saturating the growth rate of the instability has been shown in several papers (Kerswell \& Barenghi 1995; Eloy et al. 2003; Racz \& Scott 2007).

The perturbation $\mathbf{v}^{(1)}$ can be decomposed as

$$
\mathbf{v}^{(1)}=\sum_{j=1}^{2} \mathbf{v}_{j}^{(1)}=\sum_{j=1}^{2}\left(\mathbf{u}_{j}^{+} \mathrm{e}^{\mathrm{i}\left(\omega_{j} t+m_{j} \varphi+k_{j} z\right)} \pm \mathbf{u}_{j}^{-} \mathrm{e}^{\mathrm{i}\left(\omega_{j} t+m_{j} \varphi-k_{j} z\right)}\right) .
$$

where the sign is chosen such that each component $j$ has the same parity as the corresponding free Kelvin mode (a plus sign if there is a plus in (4.7)). In this case the left-hand side term in equation (4.11) has the same parity than the right-hand side terms.

Inserting the expression (4.12) for $\mathbf{v}^{(\mathbf{1})}$ and the expression (4.8) for $\mathbf{v}^{(\mathbf{0})}$ in (4.11) gives an equation for $\mathbf{v}_{\mathbf{j}}$. A solvability condition is then obtained by forming the scalar product defined by (A 10) of $\mathbf{v}_{1}$ (resp. $\mathbf{v}_{2}$ ) with this equation. It yields two coupled amplitude equations for $A_{1}$ and $A_{2}$

$$
\begin{aligned}
& \frac{\partial A_{1}}{\partial t}=\bar{\varepsilon} n_{1} A_{2}-\frac{1}{R e^{1 / 2}} s_{1} A_{1}-\frac{1}{R e} v_{1} A_{1}-\mathrm{i} q_{1} \Delta k_{1} A_{1}, \\
& \frac{\partial A_{2}}{\partial t}=\varepsilon n_{2} A_{1}-\frac{1}{R e^{1 / 2}} s_{2} A_{2}-\frac{1}{R e} v_{2} A_{2}-\mathrm{i} q_{2} \Delta k_{2} A_{2} .
\end{aligned}
$$

The terms $\partial A_{j} / \partial t$ come from the scalar product $\mathbf{v}_{j} \odot \frac{\partial}{\partial t} \mathcal{I} \mathbf{v}_{j}$.

The terms $n_{1}$ and $n_{2}$ are real and represent the interaction, through the nonlinear term of the Navier-Stokes equation, of a free Kelvin mode with the forced mode. These terms come from the scalar product $\mathbf{v}_{j} \odot\left[\mathbf{N}\left(\mathbf{v}^{(0)}, \varepsilon \mathbf{v}_{1, \omega, k}\right)+\mathbf{N}\left(\varepsilon \mathbf{v}_{1, \omega, k}, \mathbf{v}^{(0)}\right)\right]+$ c.c. They are given in Appendix B.

The coefficients $s_{1}$ and $s_{2}$ represent the surface viscous damping of the two free Kelvin modes due to Ekman boundary layers. They come from the rest of the scalar product $\mathbf{v}_{j} \odot\left(\frac{\partial}{\partial t} \mathcal{I}+\mathcal{M}\right) \mathbf{v}^{(1)}$ which almost vanishes because $\mathbf{v}_{j}$ is in the kernel of the operator $\frac{\partial}{\partial t} \mathcal{I}+\mathcal{M}$. This rest corresponds to the pressure of the free Kelvin mode $p_{j}$ times the normal velocity of $\mathbf{v}_{j}^{(1)}$ integrated over the cylinder walls. This normal velocity is given by the boundary condition (4.3) at order $\varepsilon$ and is thus the opposite of the Ekman pumping associated with the free Kelvin modes, as explained in section 4.3. This is why these terms are proportional to $R e^{-1 / 2}$. The coefficients $s_{j}$ are complex numbers with a positive real part and can be analytically calculated (see Appendix B and Kudlick 1966).

The coefficients $v_{1}$ and $v_{2}$ are real and represent the volume viscous damping of the two free Kelvin modes. They originate from the scalar product $\mathbf{v}_{j} \odot \mathcal{L} \mathbf{v}_{j}$ and can be decomposed into two parts : one proportional to $k_{j}^{2}$, another proportional to $m_{j}^{2}$ as shown in Appendix B. The free Kelvin modes with a high azimuthal wavenumber are thus strongly damped by volume viscous effects. Although these terms should appear at higher order in the asymptotic expansion, they have be taken account here to allow for the selection of modes with the largest wavelengths.

The real coefficients $q_{1}$ and $q_{2}$ represent the damping of the two free Kelvin modes by detuning effects. These terms originate from the scalar product $\mathbf{v}_{j} \odot \mathcal{M}_{\Delta} \mathbf{v}_{j}$. They can 
be analytically calculated and are given in Appendix B. These terms vanish when the two free Kelvin modes are exactly resonant.

In the following, viscous and detuning effects are gathered in a single coefficient

$$
\alpha_{j}=\frac{1}{R e^{1 / 2}} s_{j}+\frac{1}{R e} v_{j}+\mathrm{i} q_{j} \Delta k_{j},
$$

corresponding to the linear decay term.

\subsection{Growth rate of the instability}

Assuming that the amplitudes $A_{1}$ and $A_{2}$ are growing exponentially $\left(A_{j} \sim \mathrm{e}^{\sigma t}\right)$, the equation for the complex growth rate $\sigma$ is obtained by canceling the determinant of the linear system (4.13)

$$
\left(\sigma+\alpha_{1}\right)\left(\sigma+\alpha_{2}\right)=|\varepsilon|^{2} n_{1} n_{2} .
$$

This relation leads to an analytical expression for the complex growth rate. The temporal growth rate $\sigma^{\mathrm{r}}$ of the instability is simply the real part of $\sigma$. The flow is thus unstable when $\sigma^{\mathrm{r}}$ is positive for a given resonant Kelvin mode combination. The growth rate is a function of $\varepsilon$ and $R e$ only, or alternatively a function of $R o$ and $R e$.

For an exactly resonant combination (no detuning effect) and at infinite Reynolds number, the linear saturation terms $\alpha_{j}$ vanish such that the growth rate is simply given by $\sigma^{\mathrm{r}}=|\varepsilon|\left(n_{1} n_{2}\right)^{1 / 2}$. As expected (based on similarities with the elliptic instability) it is proportional to the amplitude $|\varepsilon|$ of the forced Kelvin mode. The flow is unstable if the coefficient $n_{1} n_{2}$ is positive, which is always true for Kelvin modes with dispersion relations of opposite slopes. This can be shown by looking at the signs of $n_{1}$ and $n_{2}$ (see Appendix B) and confirms the result by Fukumoto (2003) proved with energetic methods. These coeficients are given in Tables 1 and 2 for the principal exact resonances. They increase drastically when the aspect ratio decreases. This comes from the fact that the velocity of the base flow increases and it does not mean that the precessing cylinder is more unstable as it will be seen later.

When linear saturation terms are introduced $\left(\alpha_{j} \neq 0\right)$, the growth rate of the instability decreases. At infinite Reynolds numbers only detuning effects play a role and the growth rate thus takes the simple form

$$
\sigma^{\mathrm{r}}=\left[|\varepsilon|^{2} n_{1} n_{2}-\frac{1}{4}\left(q_{1} \Delta k_{1}-q_{2} \Delta k_{2}\right)^{2}\right]^{1 / 2} .
$$

Detuning effects stabilize the flow and their influence can be studied by varying the aspect ratio. Such a variation allows to observe several combinations $\left(m_{2}, l_{1}, l_{2}\right)$ which become exactly resonant (i.e. $\Delta k_{j}=0$ ) for particular values of $h$, listed in Table 1 and 2.

For an exact resonance, detuning effects vanish and the instability is damped by viscous effects only. An asymptotic expression can be obtained for large Reynolds numbers (when $\left.\operatorname{Im}\left(\alpha_{j}\right) \ll \varepsilon\left(n_{1} n_{2}\right)^{1 / 2}\right)$

$$
\sigma^{\mathrm{r}}=|\varepsilon|\left(n_{1} n_{2}\right)^{1 / 2}-\frac{\operatorname{Re}\left\{\mathrm{s}_{1}+\mathrm{s}_{2}\right\}}{2 R e^{1 / 2}}-\frac{v_{1}+v_{2}}{2 R e},
$$

where the two damping terms are due to surface and volume viscous damping. These viscous effects stabilize the flow at low amplitude $\varepsilon$ and thus allow to calculate the threshold of the instability.

\subsection{Instability threshold}

Assuming that the real part of $\sigma$ vanishes in (4.15) leads after some calculation to an expression for the amplitude $\varepsilon_{\text {crit }}$ at which the instability appears. Since this amplitude 


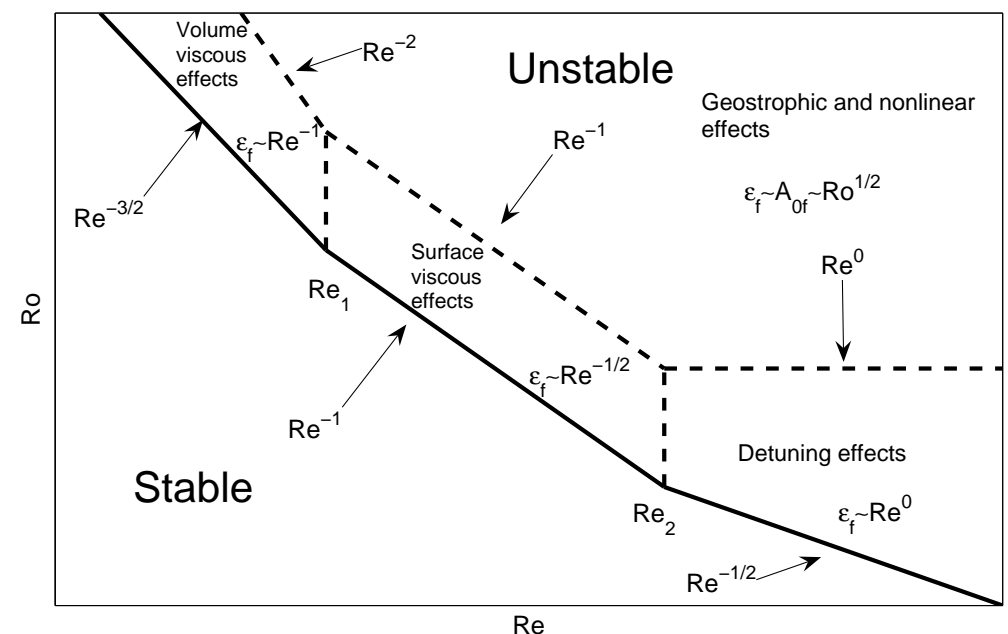

FIgURE 4. Schematic of the stability diagram in the presence of viscous and detuning effects. The solid line corresponds to the critical Rossby number at which the flow becomes linearly unstable; it exhibits three different scaling laws depending on the value of the Reynolds number compared to the critical Reynolds numbers $R e_{1}$ and $R e_{2}$ given in (4.19) and (4.22). The non-linearly saturated value $\varepsilon_{f}$ of the amplitude of the base Kelvin mode is also shown. It corresponds to the stationary solution of the non-linear system (6.3) and is calculated analytically in Appendix D.

of the forced Kelvin mode is given by Eq. (3.12), we can thus determine the critical Rossby number at which the instability appears

$$
\left|R o_{\text {crit }}\right|=\frac{1}{|f|}\left\{\frac{\alpha_{1}^{\mathrm{r}} \alpha_{2}^{\mathrm{r}}}{n_{1} n_{2}}\left[1+\left(\frac{\alpha_{1}^{\mathrm{i}}-\alpha_{2}^{\mathrm{i}}}{\alpha_{1}^{\mathrm{r}}+\alpha_{2}^{\mathrm{r}}}\right)^{2}\right]\right\}^{1 / 2}\left|\frac{s}{R e^{1 / 2}}+\frac{\mathrm{i} f}{a_{i}}\right|,
$$

where $\alpha_{j}^{\mathrm{r}}$ and $\alpha_{j}^{\mathrm{i}}$ are respectively the real and imaginary parts of $\alpha_{j}$ defined in (4.14). We recall that $f$ is the linear forcing parameter and $s$ the surface viscous parameter of the forced Kelvin mode, given in Appendix A and B. The coefficient $a_{i}$ corresponds to the inviscid amplitude of the forced Kelvin mode and is given in Appendix A. Note that equation (4.18) is not valid if $\alpha_{1}^{\mathrm{r}}+\alpha_{2}^{\mathrm{r}}=0$, which means that the inviscid threshold is not the limit of the viscous threshold for large $R e$ number. Different scalings for the critical Rossby number are obtained depending on the predominant damping effect in $\alpha_{j}$.

In the experiments, free Kelvin modes with fairly large azimuthal wavenumbers have been observed. In this case, the volume viscous damping of order $\left(m_{j}^{2}+\delta_{j}^{2}\right) R e^{-1}$ can be larger than surface viscous damping or order $R e^{-1 / 2}$ if the Reynolds number is below a critical value defined by

$$
R e_{1}=\frac{v_{1}^{\mathrm{r}} v_{2}^{\mathrm{r}}}{s_{1}^{\mathrm{r}} s_{2}^{\mathrm{r}}}\left(1+\frac{\left(s_{1}^{\mathrm{i}}-s_{2}^{\mathrm{i}}\right)^{2}}{\left(s_{1}^{\mathrm{r}}+s_{2}^{\mathrm{r}}\right)^{2}}\right)^{-1}
$$

For Reynolds numbers between 1 and $R e_{1}$, the critical Rossby number scales as $R e^{-3 / 2}$, as shown schematically in Fig. 4. A simple expression can be found in this regime

$$
\left|R o R e^{3 / 2}\right|_{\text {crit }}=\left|\frac{s}{f}\right|\left(\frac{v_{1} v_{2}}{n_{1} n_{2}}\right)^{1 / 2} .
$$

At intermediate Reynolds numbers $\left(R e \gg R e_{1}\right)$, surface viscous effects become do- 
minant. The critical Rossby number then scales as $R e^{-1}$ (see Fig. 4) and leads to an expression

$$
|R o R e|_{\text {crit }}=\left|\frac{s}{f}\right|\left\{\frac{s_{1}^{\mathrm{r}} s_{2}^{\mathrm{r}}}{n_{1} n_{2}}\left[1+\left(\frac{s_{1}^{\mathrm{i}}-s_{2}^{\mathrm{i}}}{s_{1}^{\mathrm{r}}+s_{2}^{\mathrm{r}}}\right)^{2}\right]\right\}^{1 / 2} .
$$

This regime is the most frequent in experiments and we thus give the values of the critical $|R o R e|$ for the principal resonances in Tables 1 and 2. The most unstable resonances (given by the smallest Rossby numbers) are found for the large aspect ratios $h$, although the inviscid growth rates $\left(n_{1} n_{2}\right)^{1 / 2}$ are larger at small $h$. This is due to the strong increase of $\varepsilon$ with $h$.

When the combination is not exactly resonant, detuning effects can also damp the instability. They appear at high Reynolds numbers, when surface viscous terms become smaller than detuning terms, i.e. for $R e \ll R e_{2}$ defined by

$$
R e_{2}=\frac{\left(s_{1}^{\mathrm{r}}+s_{2}^{\mathrm{r}}\right)^{2}+\left(s_{1}^{\mathrm{i}}-s_{2}^{\mathrm{i}}\right)^{2}}{\left(q_{1} \Delta k_{1}-q_{2} \Delta k_{2}\right)^{2}} .
$$

In this regime, the critical Rossby number still depends on the Reynolds number since the forced Kelvin mode scales as $R e^{1 / 2}$. The critical Rossby number thus scales as $R e^{-1}$ and leads to a simple expression

$$
\left|R o R e^{1 / 2}\right|_{\text {crit }}=\left|\frac{s}{f}\right| \frac{\left(s_{1}^{\mathrm{r}} s_{2}^{\mathrm{r}}\right)^{1 / 2}}{s_{1}^{\mathrm{r}}+s_{2}^{\mathrm{r}}} \frac{\left|q_{1} \Delta k_{1}-q_{2} \Delta k_{2}\right|}{\left(n_{1} n_{2}\right)^{1 / 2}} .
$$

It can be noted that this expression is valid for $R e$ asymptoting to infinity and takes into account the values of the viscous coefficients $s_{j}$. It is different from the value that would be obtained at infinite Reynolds number by assuming $\sigma^{\mathrm{r}}=0$ in (4.16), but the two theories give identical threshold when $s_{1}=s_{2}$.

\section{Discussion}

In this section we discuss the general properties of the precessional instability as a function of the dimensionless parameters $R e, h$ and $\omega$ and we compare these predictions to experimental results for a particular aspect ratio. We limit our study to the instabilities driven by the first forced Kelvin mode.

\subsection{Prediction of the unstable modes}

In our previous paper (Lagrange et al. 2008), we have observed experimentally that the unstable flow exhibits two free Kelvin modes with azimuthal wavenumbers 5 and 6 at the first resonance of the first Kelvin mode ( $h=1.62$ and $\omega=1.18$ ). Their experimental axial and instantaneous vorticity fields are plotted in figures $5(a)$ and $5(b)$. They correspond exactly to the free Kelvin modes predicted theoretically for this aspect ratio. Indeed, the resonant combination $(6,1,1)$ is more unstable than any other combinations (with different azimuthal wavenumbers $m_{j}$ and branch numbers $l_{j}$ ). The theoretical axial vorticity fields of the free Kelvin modes are shown in figures $5(c)$ and $5(d)$ and look very similar to the experimental fields. They both have one ring of 10 (or 12) alternate vortices meaning that they correspond to the first branch of the dispersion relations $\left(l_{1}=l_{2}=1\right)$ for $m_{1}=5$ and $m_{2}=6$. Furthermore, the radial position of these vortices seems to be well predicted : the maximum of the azimuthal average vorticity is located theoretically at $r=0.80$ and experimentally at $r \approx 0.83$ for the second mode with $m_{2}=6$. The agree- 


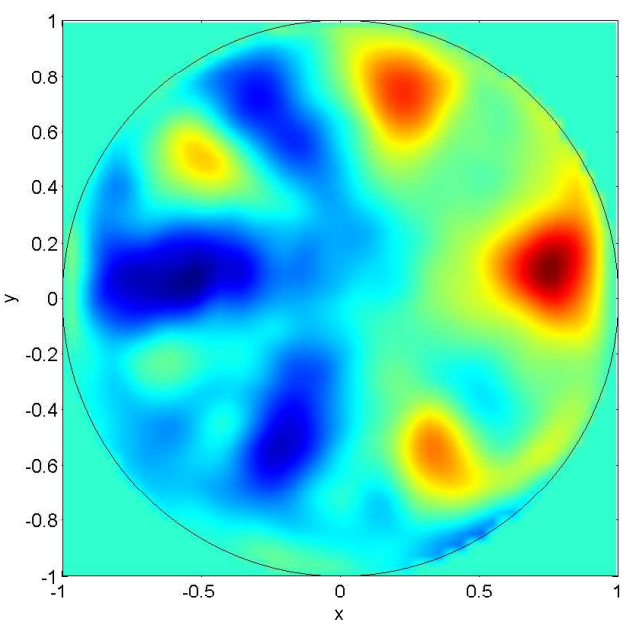

(a)

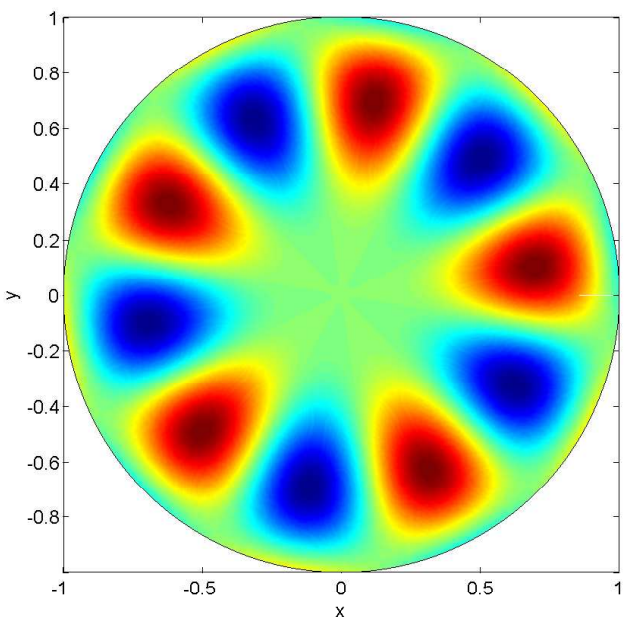

(c)

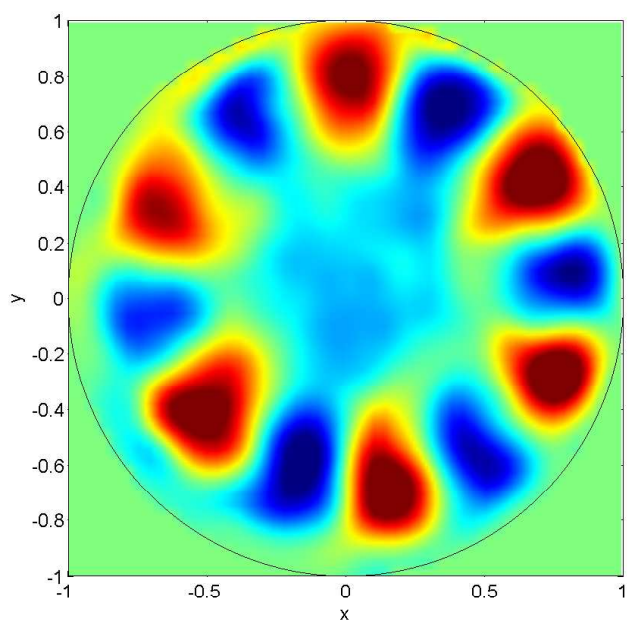

(b)

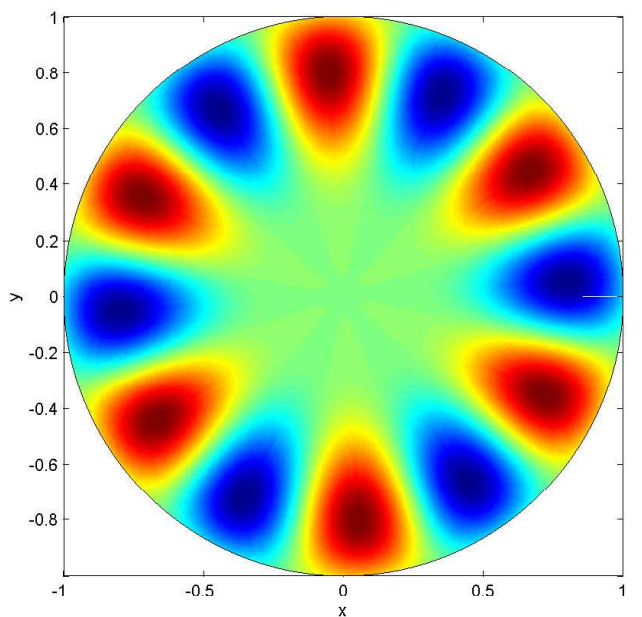

(d)

Figure 5. Experimental (a, b) and theoretical (c, d) axial and instantaneous vorticity fields of the free Kelvin modes $m_{1}=5$ and $m_{2}=6 . h=1.62, \omega=1.18$ and $R o=-0.0031$. (a) $R e=6000$. (b) $R e=6500$.

ment is not as good for the first mode with $m_{1}=5(r=0.70$ theoretically compared to $r \approx 0.57$ experimentally) because of the presence of the forced Kelvin mode $(m=1)$.

To better compare the structure of the unstable modes we have plotted in figure 6 the theoretical and the experimental averaged radial and azimuthal velocities of the free Kelvin modes as a function of $r$. They have been obtained by taking the azimuthal and the temporal average of the velocity fields and they are given in arbitrary units. The averaged radial velocity profiles of the free Kelvin modes are very similar and look like bell-shaped profiles. Because the two free Kelvin modes satisfy a condition of no outward flow at $r=1$, their averaged radial velocities vanishes at $r=1$. The averaged azimuthal velocity profiles are more complicated. The averaged azimuthal velocity profile of the free Kelvin mode $m_{1}=5$ shows two extrema while for the mode $m_{2}=6$, it shows one 


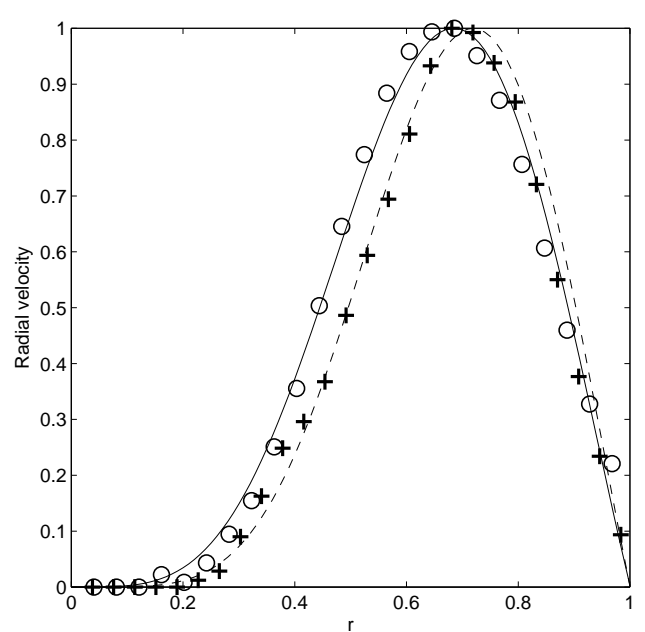

(a)

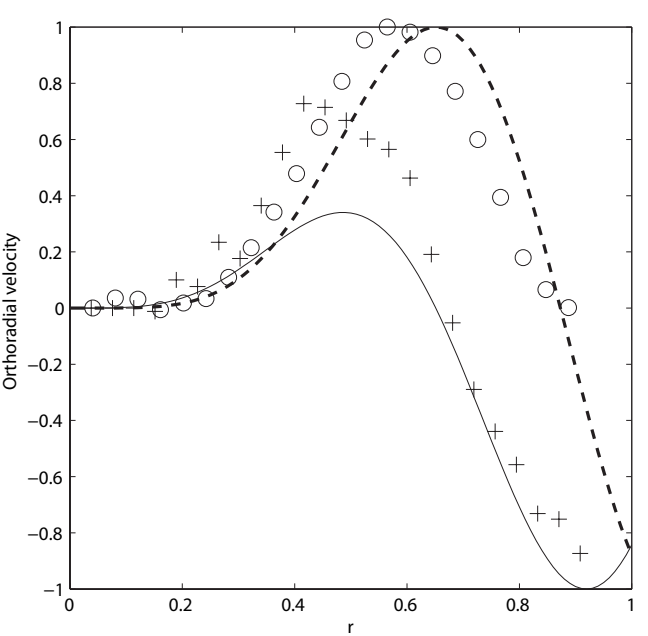

(b)

Figure 6. Averaged radial (a) and azimuthal (b) velocity profiles of the free Kelvin modes $m_{1}=5$ ( + , solid line), and $m_{2}=6$ ( $\circ$, dashed line), in arbitrary units. Symbols represent experimental data and lines are issued from the theory. $h=1.62, \omega=1.18, R e=6000$ for $m_{1}=5 ; R e=6500$ for $m_{2}=6$.

extremum. Experiments roughly confirm these two different averaged azimuthal velocity profiles.

Finally, theory also predicts that the free Kelvin mode $m_{1}=5$ (resp. $m_{2}=6$ ) has an axial vorticity which is a sine (resp. cosine) function of the altitude $z$. This parity is confirmed by experiments since the free Kelvin mode $m_{1}=5$ (resp. $m_{2}=6$ ) has been observed at $z=0$ (resp. $z=h / 4$ ).

These experimental observations confirm the mode predicted by the linear stability analysis and therefore validate the mechanism of triadic resonance proposed in this paper.

\subsection{Prediction of the growth rate}

In this section, we compare the theoretical growth rate $\sigma^{\mathrm{r}}$, with the experimental results. We restrict our analysis to the first resonance of the first Kelvin mode for $h=1.62$, such that there is no detuning effects.

Figure 7 represents the evolution of the growth rate as a function of the forcing. In the inviscid case, the theory gives a constant value $\sigma^{\mathrm{r}} /|\varepsilon|=\left(n_{1} n_{2}\right)^{1 / 2}$ represented by a solid line. When surface viscous effects are added, it can be easily shown (dividing (4.15) by $|\varepsilon|^{2}$ ) that the rescaled growth rate $\sigma^{\mathrm{r}} /|\varepsilon|$ is a function of $|\varepsilon| R e^{1 / 2}$ only. This is why we have plotted the growth rate in these coordinates. This viscous prediction is plotted as a dash-dotted line in Fig. 7. The growth rate increases with the forcing and tends toward the inviscid growth rate at infinite Reynolds number. It vanishes at a specific value of $|\varepsilon| R e^{1 / 2}=0.843$ corresponding to the critical Rossby number (given in Table 1 ). This prediction is only valid for Reynolds numbers large compared to $R e_{1}$. Below this Reynolds number, volume viscous effects must be taken into account. The theoretical prediction with all viscous terms is plotted as a dashed line for $R e=6000$ and as a dotted line for $R e=1000$. They show the same trend but remain weaker.

Experimental measurements of the growth rate are also plotted as symbols on the same graph. As expected, experimental measurements are below the growth rate with surface viscous terms only. They are represented as filled (resp. open) symbols when their 


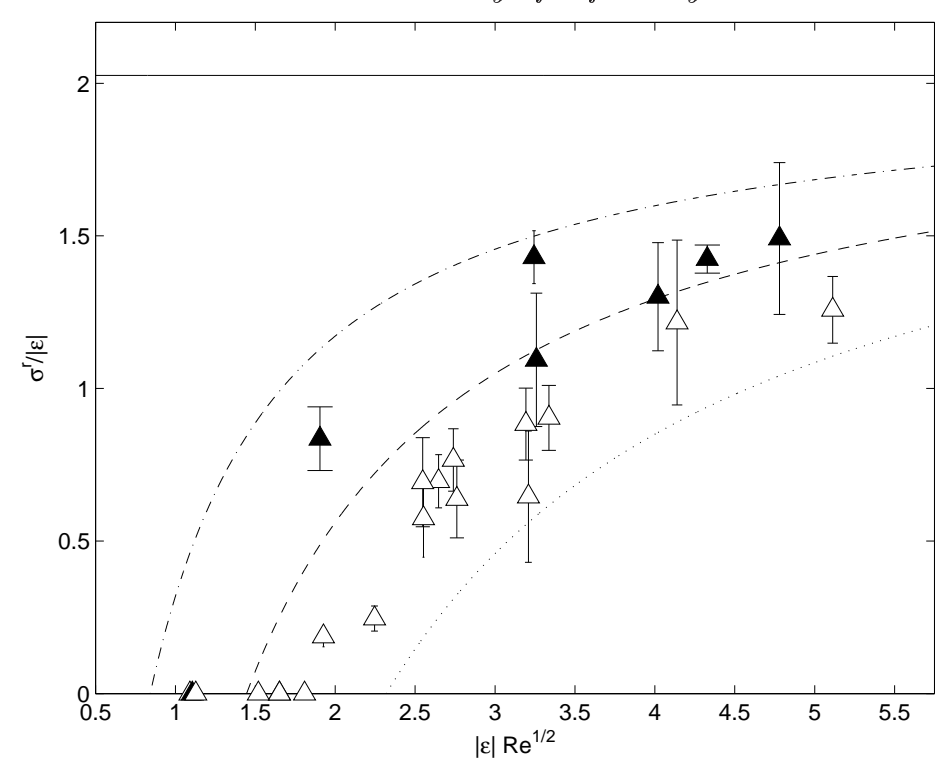

Figure 7. Growth rate $\sigma^{\mathrm{r}} /|\varepsilon|$ of the precessional instability for $h=1.62$ and $\omega=1.18$. The inviscid prediction is represented by the horizontal solid line. The dash-dotted line is the viscous prediction without volume viscous effects. The dashed line is the full viscous prediction for $R e=6000$. The dotted line is the viscous prediction for $R e=1000$. Filled symbols $(\mathbf{\Delta})$ correspond to experiments with $6000<R e<24400$. Open symbols $(\triangle)$ correspond to experiments with $1000<R e<6000$. The error bars correspond to the standard deviation on the fitting parameter.

Reynolds number is larger (resp. smaller) than the Reynolds number of the dashed line $(R e=6000)$. The symbols always fall within the correct band of growth rate, which is a good confirmation of the theoretical calculation. A quantitative comparison indicates that the measured growth rate is never farther than $50 \%$ from the theoretical prediction.

Experimentally, the growth rate was measured by an exponential fit of the amplitude of the free Kelvin mode $m_{1}=5$ at the onset of the instability. Note that the instability can appear before the amplitude $|\varepsilon|$ of the forced Kelvin mode has reached its stationary value given by equation (3.12). So here $|\varepsilon|$ corresponds to the measured amplitude of the first Kelvin mode at the onset of instability and not the value given by (3.12).

\subsection{Critical Rossby number as a function of Re}

Figure 8 represents the critical Rossby number $R o_{\text {crit }}$ as a function of $R e$ when the first Kelvin mode is forced at its first resonance. The prediction is issued from equation (4.18) and is represented by a solid line which divides the $(R e, R o)$-plane into a stable domain and an unstable domain. Stable experiments are represented by circles and unstable experiments are represented by black symbols. These experiments were done on a very large range of precessing angle $\left(0.25^{\circ}\right.$ to $\left.10^{\circ}\right)$ and Reynolds number $\left(10^{3}\right.$ to $\left.5 \times 10^{4}\right)$. It is striking to see such an agreement between experiments and theory with no fitting parameter in the theory. However, there are some slight discrepancies at high Rossby numbers. They may come from non-linear effects in the base flow which arise because the amplitude of the forced Kelvin mode $\varepsilon \sim R o R e^{1 / 2}$ is no longer a small parameter. Note also that the uncertainty is larger at small Rossby number because the precessing angle (equal to $0.25^{\circ}$ ) becomes comparable to the uncertainty of $\pm 0.1^{\circ}$.

Because the Reynolds number is varied over a large range (one decade), the experiments 


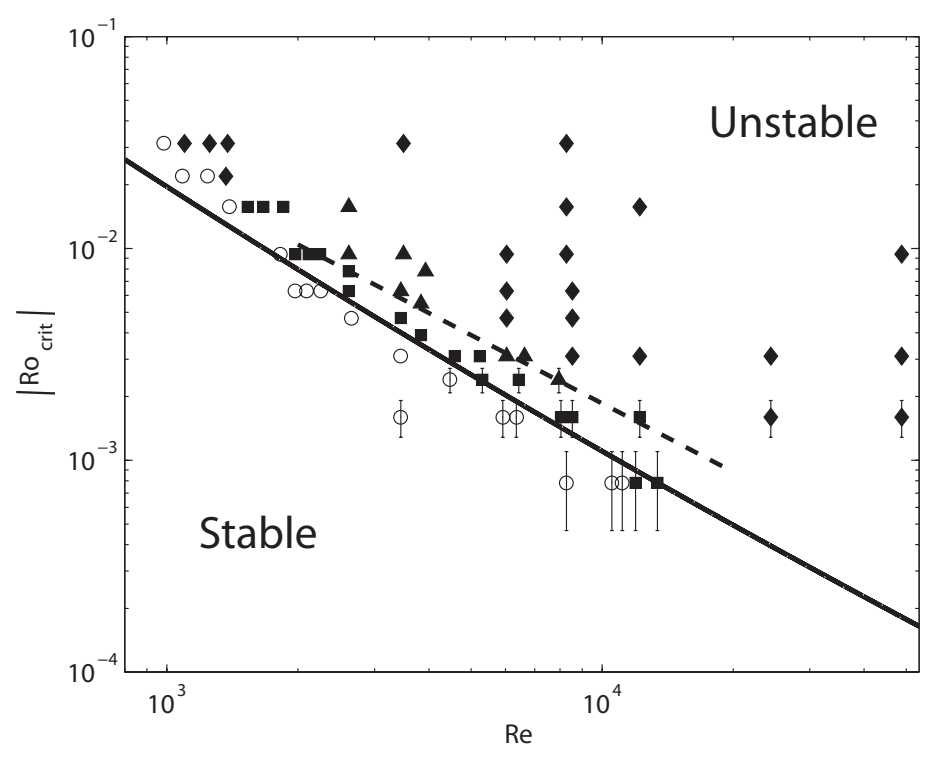

Figure 8. Stability diagram of the flow inside a precessing cylinder for $h=1.62$ and $\omega=1.18$. The stable and unstable domain are separated by the solid line which corresponds to the prediction (4.18). Open symbols (o) represent stable experiments and filled symbols represent unstable experiments. Unstable experiments can be either stationary (ם), intermittent $(\boldsymbol{\Delta})$ or turbulent $(\downarrow)$. The error bars come from the uncertainty in the measurement of the precessing angle. The dashed line corresponds to the transition from a stationary to an intermittent flow in the weakly non-linear model.

allow to confirm the scalings for the critical Rossby number. As was shown earlier, at moderate Reynolds number, ( $\left.R e \ll R e_{1}=3000\right)$ the Rossby number scales as $R e^{-3 / 2}$. At large Reynolds numbers $\left(R e \gg R e_{1}=3000\right.$, the Rossby number scales as $R e^{-1}$.

\subsection{Critical RoRe number as a function of $h$}

Now that the theory has been validated experimentally by the two previous sections, we use these predictions to study the general properties of the precessional instability as the parameters $h$ and/or $\omega$ are varied. In this section, the aspect ratio is varied over a large range, from 0.2 to 10 . However, the frequency $\omega$ always corresponds to the first resonance of the first Kelvin mode $\omega_{1,1}$.

Figure 9 shows the evolution of the threshold as a function of $h$. Several bands of instability corresponding to different resonant Kelvin mode combinations $\left(m_{2}, 1,1\right)$ are observed. Each band reaches a minimum when the combination is exactly resonant, i.e. when detuning effects vanish. The most unstable combination (i.e. minimum threshold) is the $(6,1,1)$ resonant combination for $h=1.62$, studied experimentally in this paper.

We have seen in section 4.7 that, when volume viscous effects are neglected, the stability threshold only depends on the product RoRe. This is why we have plotted the threshold as $|R o R e|$ in figure 9, such that the prediction without volume viscous effects is independent of the Reynolds number and plotted as a symbol for each exactly resonant combination. The gap between the symbol the corresponding solid line indicate the influence of the volume viscous effects at $R e=6500$.

The combinations $(2,1,1)$ and $(3,1,1)$ are special because they are not close to an exact resonance (which ensures a small detuning) although they are the most unstable for small aspect ratios. 


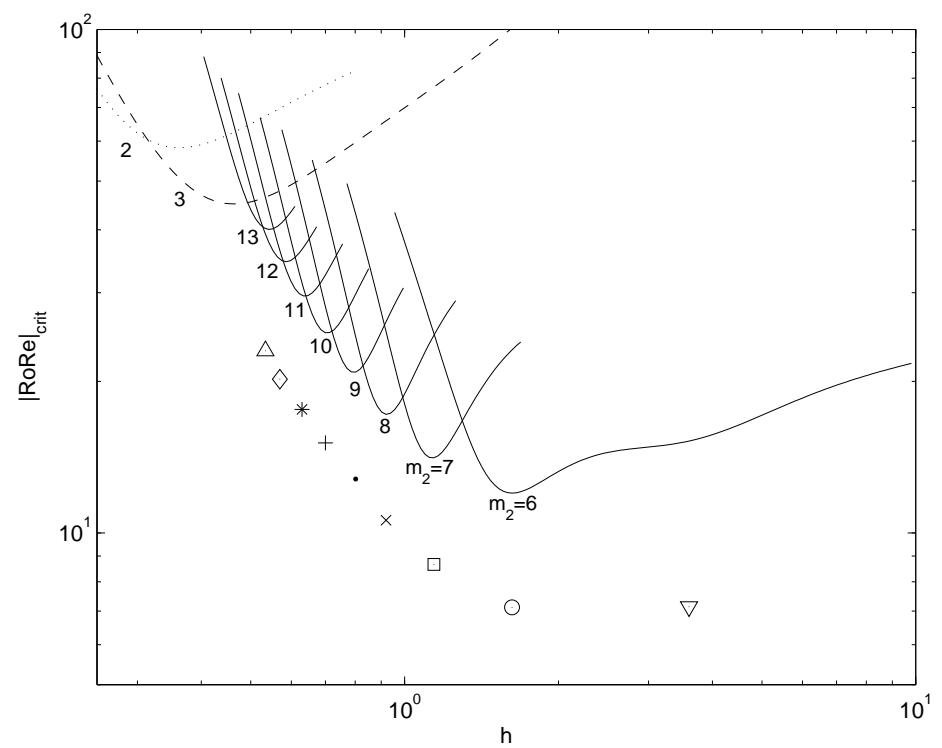

Figure 9. Critical $|R o R e|$ number of the most unstable modes as a function of $h$ for the first resonance of the first Kevlin mode $\left(\omega=\omega_{1,1}\right)$. Each solid line corresponds to the full theory at $R e=6500$ for a resonant combination $\left(m_{2}, 1,1\right)$. Symbols correspond to the predictions without detuning and without volume viscous effects (thus valid at any Reynolds number, see Table 1) for the same combinations with $m_{2}=6(\circ, \nabla), m_{2}=7(\square), m_{2}=8(\times), m_{2}=9(\bullet), m_{2}=10$ $(+), m_{2}=11(*), m_{2}=12(\diamond), m_{2}=13(\triangle)$. The dashed line (resp. dotted line) corresponds to a combination $(3,1,1)$ (resp. $(2,1,1)$ ) which can not be exactly resonant.

Finally note that some resonant Kelvin mode combinations $\left(m_{2}, 2,2\right)$ (not represented in figure 9) can be more unstable than the combinations $\left(m_{1}, 1,1\right)$ represented in very small intervals of aspect ratios when $h<1$.

\subsection{Critical Rossby number as a function of $\omega$}

In this section the aspect ratio and the Reynolds number are fixed and the frequency $\omega$ is varied around the first resonance of the first Kelvin mode. Figure 10 represents the critical Rossby number as a function of $\omega$, for $h=1.62$ and $R e=6500$. Several bands of instability corresponding to different azimuthal wavenumbers $m_{2}=7$ (dotted line), $m_{2}=6$ (solid line) and $m_{2}=5$ (dashed line) are predicted. This is due to the change of $\omega$ which allow to couple different Kelvin modes (since $\omega_{2}-\omega_{1}=\omega$ ). These predictions are well confirmed by PIV measurements which reveal the correct azimuthal wavenumber. Moreover, the theoretical threshold is in very good agreement with experiments.

The most unstable combination is the $(6,1,1)$ mode and is obtained close to $\omega=$ 1.18. This can be easily understood because this is where the amplitude $\varepsilon$ of the forced Kelvin mode is maximum (at its resonance). Around the resonance, the amplitude of the forced Kelvin mode decreases as $1 /\left(\omega-\omega_{1,1}\right)$, which lead to weaker instabilities for the combinations $(5,1,1)$ and $(7,1,1)$.

This proves that the easiest way to trigger an instability is to force the base flow at a resonance frequency. For example, here, a precessing angle of $\theta \approx 0.5^{\circ}$ is sufficient to observe the $(6,1,1)$ resonant Kelvin mode combination. Outside of the resonant frequency it is necessary to have a minimum precessing angle of $\theta \approx 7^{\circ}$ (resp. $\theta \approx 2^{\circ}$ ) to observe the $(7,1,1)$ (resp. $(5,1,1))$ resonant Kelvin mode combination at the same Reynolds number. 


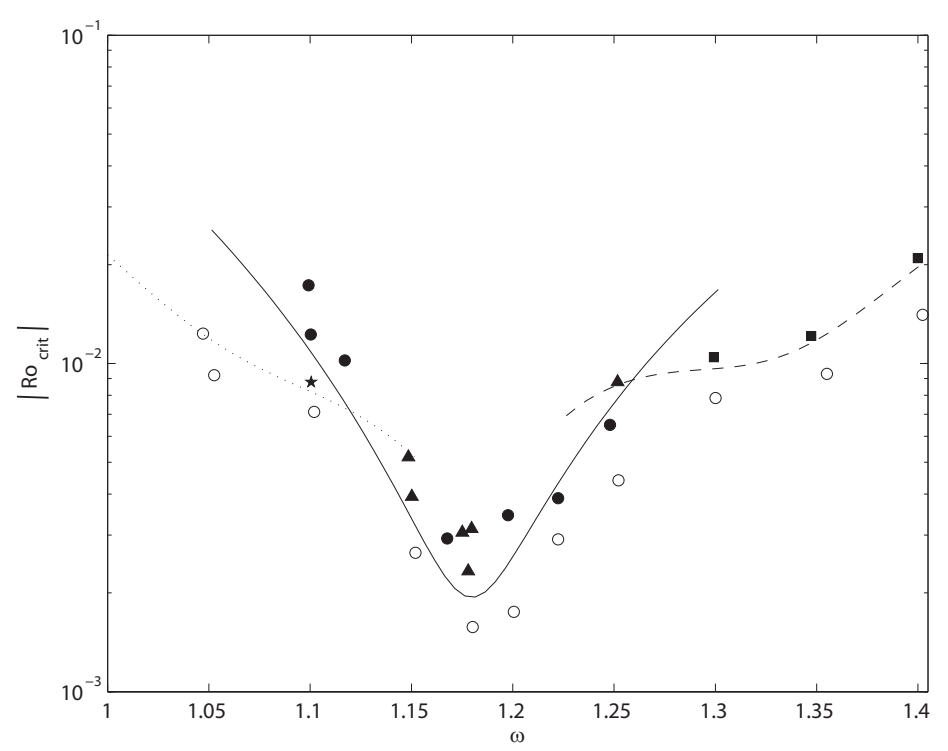

Figure 10. Critical Rossby number as a function of $\omega$. Resonant Kelvin mode combinations $(7,1,1)$ (dotted line), $(6,1,1)$ (solid line) and $(5,1,1)$ (dashed line) are observed. Stable experiments are represented by circles ( $\circ$ ) and unstable experiments by black symbols. PIV experiments are represented by a black star $(\star)$ for the $(7,1,1)$ combination, black triangles $(\mathbf{\Lambda})$ for the $(6,1,1)$ combination and black squares $(\boldsymbol{\square})$ for the $(5,1,1)$ combination. Black circles $(\bullet)$ correspond to unstable experiments visualized by using Kalliroscope particles. $h=1.62$, $R e=6500$.

\subsection{Critical Rossby number as a function of $h$ and $\omega$}

In this section we study the general problem of the precessional instability at a given Reynolds number when both the aspect ratio and the frequency are varied. We consider only instabilities triggered by the first forced Kelvin mode. The critical Rossby number in the plane $(h, \omega)$ is represented in figure 11 . It was obtained numerically by calculating the analytical coefficients of the amplitude equations (4.13) for various azimuthal wavenumbers ( $m_{2}=1$ to 15$)$ and for various branches $\left(l_{j}=1\right.$ to 5$)$ on a very fine mesh of the plane $(h, \omega)$. This calculation took about a month on a standard computer.

On this figure, the most unstable regions correspond to the dark areas, i.e. where the critical Rossby number is small. There are two unstable regions, which are located around the resonances of the first Kelvin mode, represented by white dashed lines. As explained in the previous section, this can be easily understood because the amplitude of the forced Kelvin mode $\varepsilon$ is maximum there.

The black thin lines separate different resonant Kelvin mode combinations with azimuthal wavenumbers $m_{2}=5$ to 8 . It is surprising to see that each combination creates a band which is almost parallel to the resonance curve. This explains why there are only a few combinations which are resonant (here only 4 combinations) when varying the aspect ratio. The combination $(6,1,1)$ is the most unstable one because it is centered on the resonance curve almost over the whole range of aspect ratios considered here. It is curious to see that it is exactly resonant twice (for $h=1.62$ and $h=3.6$ ).

At higher Reynolds numbers, other combinations might become more unstable and thus create other bands of instabilities in between these combinations. However, one can have a rough idea of the stability diagram at any Reynolds number by simply assuming 


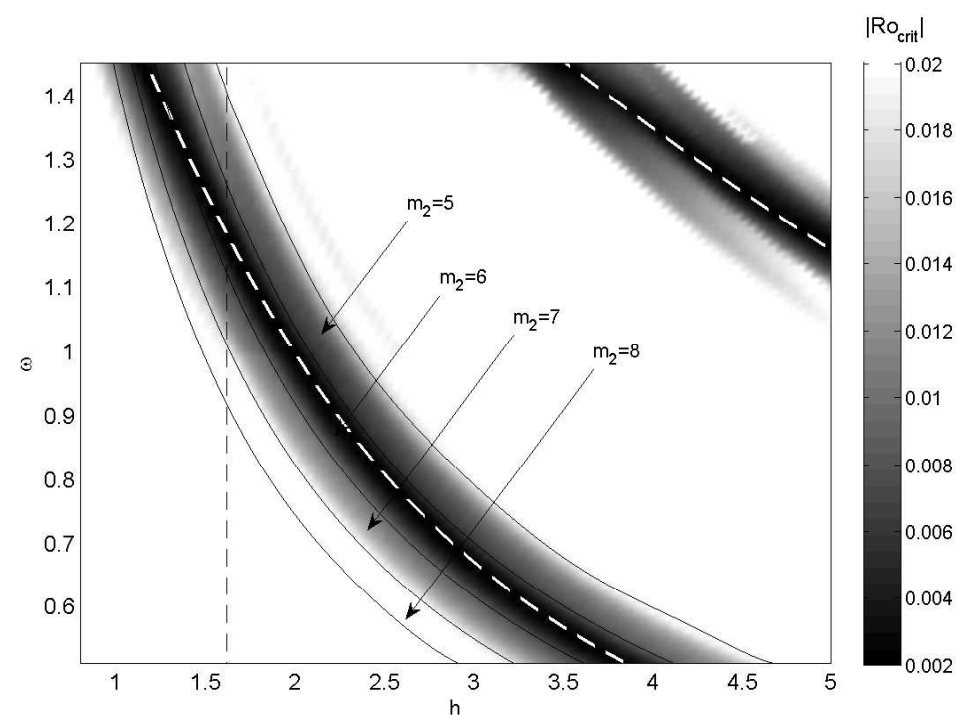

Figure 11. Critical Rossby number as a function of $h$ and $\omega$, by considering that the first Kelvin mode is forced by precession. The resonant Kelvin mode combinations $(5,1,1),(6,1,1)$, $(7,1,1),(8,1,1)$ are observed. The white dashed lines correspond to the first (lower left) and second (upper right) resonance of the first Kelvin mode. Fig. 10 corresponds to the vertical dashed line at $h=1.62$. $R e=6500$.

that the critical Rossby number is inversely proportional to the Reynolds number (as it is the case when surface viscous terms are considered).

Note that the previous figures correspond to the critical Rossby number along the white dashed line (for Fig. 9) and along the black dashed line (for Fig. 10). Note also that the combinations represented in figure 11 always satisfy $k_{2}-k_{1}=k$ (combinations satisfying $k_{1}-k_{2}=k$ are always more stable).

\section{Weakly nonlinear theory}

This section is devoted to the prediction of the instability saturation by nonlinear effects. The analysis will be restricted to the most unstable case, i.e. the exact resonance of the first Kelvin mode $(h=1.62, \omega=1.18)$.

\subsection{Geostrophic flow}

Experiments have shown that the unstable Kelvin modes $m_{1}=5$ and $m_{2}=6$ mentioned previously give rise to a mode with a cylindrical symmetry which corresponds to a stationary azimuthal velocity field. This geostrophic Kelvin mode appears at order $O\left(A_{j}^{2} R e^{-1 / 2}\right)$. It is due to the nonlinear interaction in the Ekman layers of the unstable Kelvin modes with their respective viscous flows (see Meunier et al. 2008). The role of the geostrophic mode is essential in the weakly nonlinear analysis because it saturates the amplitude of the resonant and the unstable Kelvin modes. Note that the nonlinear interaction of a Kelvin modes with itself has also a saturating effect. However, in our experiments, this saturating effect is less significative than the saturation due to the geostrophic mode.

Finding an analytical expression of the geostrophic flow is a very complex problem. To avoid this difficulty and to keep in the analysis the fewest number of mode possible, 
an empirical formulation has been used for the geostrophic mode based on experimental measurements. For $h=1.62$ and $\omega=1.18$ (i.e. first resonance of the first Kelvin mode) experiments have shown that the free Kelvin modes $m_{1}=5$ and $m_{2}=6$ lead to a geostrophic Kelvin mode whose profile is close to the profile of the free Kelvin mode

$$
\mathbf{v}_{0}=-J_{5}\left(d_{2} r\right) \mathbf{u}_{\varphi}
$$

where $\mathbf{u}_{\varphi}$ is the orthoradial unit vector and $J_{5}$ is the Bessel function of the first kind. The parameter $d_{2}$ is the second root of $J_{5}$ (i.e. $d_{2}=12.339$ ). We will assume that this geostrophic mode is added to the perturbation with an amplitude $A_{0}$.

\subsection{Weakly nonlinear amplitude equations}

Adding this geostrophic mode leads to a total flow

$$
\mathbf{v}=\varepsilon \mathbf{v}_{1, \omega, k}+A_{1} \mathbf{v}_{1}+A_{2} \mathbf{v}_{2}+A_{0} \mathbf{v}_{0}+\text { o.t. }
$$

We recall that $\varepsilon$ is the amplitude of the forced Kelvin mode $\mathbf{v}_{1, \omega, k}$ given by (3.9). Vectors $\mathbf{v}_{1}$ and $\mathbf{v}_{2}$ are the two free Kelvin modes of the triadic resonance, whose amplitudes are $A_{1}$ and $A_{2}$. These vectors are given by equation (4.7). The notation o.t. stands for 'other terms' and includes the Ekman layer pumping flows and the non-resonant Kelvin modes.

Inserting (6.2) into the Navier-Stokes equation (3.5) and forming the scalar product of this equation with $\mathbf{v}_{1, \omega, k}, \mathbf{v}_{1}, \mathbf{v}_{2}$ and $\mathbf{v}_{0}$, leads to the following weakly nonlinear amplitude equations

$$
\begin{aligned}
\frac{\partial \varepsilon}{\partial t} & =\mathrm{i} f R o-\alpha \varepsilon-\mathrm{i} \xi A_{0} \varepsilon+\lambda \overline{A_{1}} A_{2}, \\
\frac{\partial A_{1}}{\partial t} & =\bar{\varepsilon} n_{1} A_{2}-\alpha_{1} A_{1}-\mathrm{i} \xi_{1} A_{0} A_{1}-\mathrm{i} \sigma_{1}\left|A_{1}\right|^{2} A_{1}, \\
\frac{\partial A_{2}}{\partial t} & =\varepsilon n_{2} A_{1}-\alpha_{2} A_{2}-\mathrm{i} \xi_{2} A_{0} A_{2}-\mathrm{i} \sigma_{2}\left|A_{2}\right|^{2} A_{2}, \\
\frac{\partial A_{0}}{\partial t} & =\frac{1}{R e^{1 / 2}}\left(-\frac{2}{h} A_{0}+\chi_{1}\left|A_{1}\right|^{2}+\chi_{2}\left|A_{2}\right|^{2}\right) .
\end{aligned}
$$

The first equation (6.3a) governs the temporal evolution of the forced Kelvin mode amplitude $\varepsilon$. All the coefficients of this equation can be calculated analytically. The first right hand-side term is the forcing due to the precessional motion, with a linear forcing parameter $f$ given in Appendix A. The second term is due to the surface viscous damping of the amplitude of the forced Kelvin mode and is thus proportional to $\alpha=s / R e^{1 / 2}$ ( $s$ being given in Appendix B). The third term comes from the nonlinear interaction of the forced Kelvin mode with the geostrophic mode. The parameter $\xi$ can be calculated knowing the radial profile of the geostrophic mode given by (6.1) (see Appendix C). The last term is the saturating term due to the nonlinear interaction between the two free Kelvin modes with a parameter $\lambda$ given in Appendix C. In the absence of instability, the amplitude $\varepsilon$ grows and saturates due to viscous damping at a value if $R o / \alpha$.

Equations $(6.3 b)$ and $(6.3 c)$ represent the temporal evolutions of the free Kelvin mode amplitudes $A_{1}$ and $A_{2}$. All the parameters of these equations can also be calculated analytically. These equations are similar to the linear amplitude equations $(4.13 a)$ and $(4.13 b)$ with nonlinear effects added. The first right hand-side term of these equations represents the nonlinear interaction of the forced Kelvin mode with a free Kelvin mode $\left(n_{1}\right.$ and $n_{2}$ are given in Appendix B). The surface, volume viscous and detuning parameters are included in $\alpha_{1}$ and $\alpha_{2}$ given by equation (4.14). The third term of these equations was not considered before and comes from the nonlinear interactions of the free Kelvin modes with the geostrophic mode (parameters $\xi_{j}$ are given in Appendix C). The last 


\begin{tabular}{|c|c|c|c|c|c|}
\hline & $\begin{array}{c}f \\
0.226\end{array}$ & $\begin{array}{c}s \\
6-0.42 \mathrm{i}\end{array}$ & $\begin{array}{c}\xi \\
0.165\end{array}$ & $\begin{array}{c}\lambda \\
6.503\end{array}$ & \\
\hline $\begin{array}{c}n_{1} \\
-1.672\end{array}$ & $1.61-0.06 \mathrm{i}$ & $\begin{array}{c}v_{1} \\
87.16\end{array}$ & $\begin{array}{c}\xi_{1} \\
-0.066\end{array}$ & $\begin{array}{c}\sigma_{1} \\
-83.784\end{array}$ & $\begin{array}{c}\chi_{1} \\
10000\end{array}$ \\
\hline $\begin{array}{c}n_{2} \\
-2.456\end{array}$ & $1.81-0.13 \mathrm{i}$ & $\begin{array}{c}v_{2} \\
102.68\end{array}$ & $\begin{array}{c}\xi_{2} \\
-0.365\end{array}$ & $\begin{array}{c}\sigma_{2} \\
48.987\end{array}$ & $\begin{array}{c}\chi_{2} \\
10000\end{array}$ \\
\hline
\end{tabular}

TABLE 3. Numerical values for the parameters appearing in the nonlinear amplitude equations (6.3). For these values, $h=1.62$ and $\omega=1.18$ (first resonance of the first Kelvin mode). They correspond to the $(6,1,1)$ resonant combination for the case $k_{2}-k_{1}=k$.

term of equations $(6.3 b)$ and $(6.3 c)$ correspond to the interactions of a free Kevin mode with itself (parameters $\sigma_{j}$ are given in Appendix C).

Equation (6.3d) describes the evolution of the geostrophic Kelvin mode amplitude $A_{0}$. The first right hand-side term represents the surface viscous damping of the geostrophic Kelvin mode. Note that for this mode volume viscous effects have been neglected relative to surface viscous effects. The second and the third terms of this equation represent the nonlinear interaction of each free Kelvin mode with its Ekman pumping flow. The parameters $\chi_{1}$ and $\chi_{2}$ are difficult to calculate analytically. Because modes $m_{1}=5$ and $m_{2}=6$ are similar we shall assume that $\chi_{1}$ and $\chi_{2}$ are equal. Their value will be adjusted to fit the experimental data.

We thus have a system of four nonlinear amplitude equations with only one fitting parameter $\chi_{1}=\chi_{2}$. The numerical values of the parameters are given in Table 3 . We will analyse in the following the properties of this dynamical system and compare them to experimental results.

\subsection{Nonlinear evolution of the amplitudes}

A numerical solution of the system (6.3) is shown in figure 12(a). This figure represents the temporal evolution of the amplitude of the free Kelvin mode $m_{1}=5$ for three Reynolds numbers. This corresponds to the most unstable case of the first resonance of the first Kelvin mode $(h=1.62, \omega=1.18)$, for which the numerical values of the parameters are given in Table 3 .

In the stable regime $(R e=3500, \circ)$, the amplitude of the first Kelvin mode $A_{1}$ remains equal to 0 . The amplitudes of the second Kelvin mode $A_{2}$ and the geostrophic mode $A_{0}$ are equally null. However, the amplitude $\varepsilon$ of the forced Kelvin mode grows and saturates at the viscous value given by equation (3.12) with $a_{i}=\infty$.

Just above the instability threshold ( $R e=4500$, solid line) the amplitude $A_{1}$ grows and then saturates at a given value. This unstable and stationary flow thus exhibits a fixed point that will be noted $\left(\varepsilon_{f}, A_{1 f}, A_{2 f}, A_{0 f}\right)$. Numerically, the instability saturation is found to be caused mainly by the nonlinear the term $\mathrm{i} \xi \mathrm{A}_{0} \varepsilon$. In other words, the geostrophic mode is responsible of the saturation because it reduces the amplitude of the forced Kelvin mode.

At higher Reynolds number ( $R e=6000$, dashed line), the flow is still unstable but we also observe that the amplitudes are now oscillating, even in the permanent regime. This means that the fixed point $\left(\varepsilon_{f}, A_{1 f}, A_{2 f}, A_{0 f}\right)$ has become unstable and the flow is said to be unstable and intermittent. Such a flow can be understood with the following reasoning. First, the amplitudes $A_{1}$ and $A_{2}$ grow exponentially due to the terms $\bar{\varepsilon} n_{1} A_{2}$ and $\varepsilon n_{2} A_{1}$ in equations $(6.3 b)$ and $(6.3 c)$. Then, the geostrophic mode is forced through the coefficients $\chi_{1}$ and $\chi_{2}$ appearing in equation (6.3d). This geostrophic mode grows 


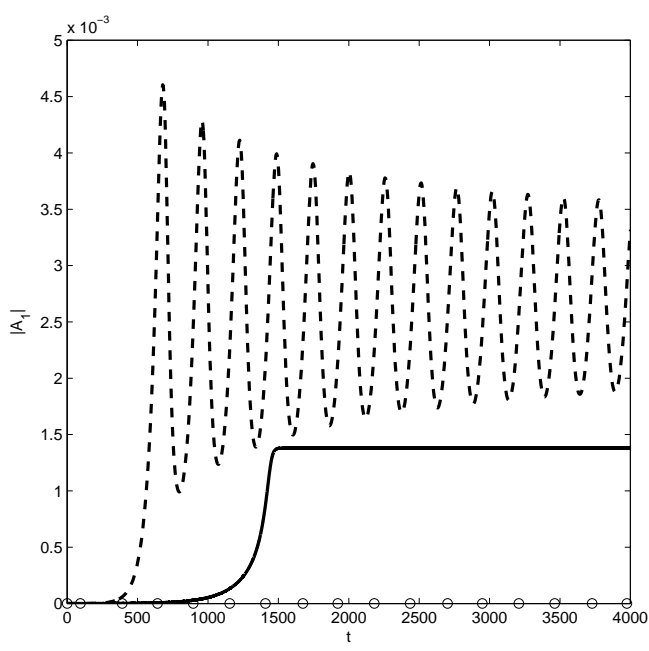

(a)

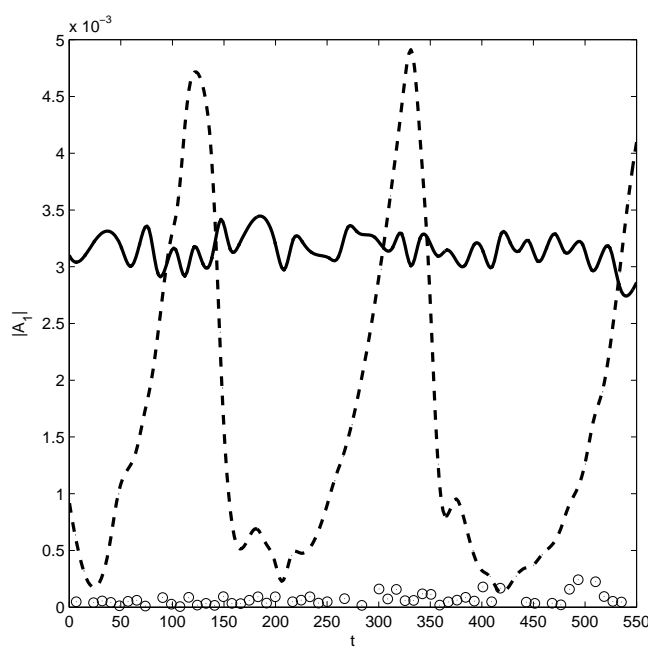

(b)

Figure 12. Amplitude of the free Kelvin mode $m_{1}=5$ as a function of the dimensionless time $t$ showing the three different regimes of the nonlinear evolution of the instability. (a) Numerical simulation. (b) Experimental results. It is obtained at $R e=3500$ (o), $R e=4500$ (solid line) and $R e=6000$ (dashed line). The frequency of acquisition $f$ and the time delay $\Delta t$ between PIV pairs are $f=0.2 \mathrm{~Hz}, \Delta t=50 \mathrm{~ms}(\circ) ; f=0.222 \mathrm{~Hz}, \Delta t=45 \mathrm{~ms}$ (solid line) ; $f=0.333$ $\mathrm{Hz}, \Delta t=30 \mathrm{~ms} . h=1.62, \omega=1.18, R o=-0.0031$.

slowly in time (since its characteristic time is of the order of $h R e^{1 / 2}$ ), which creates a delay to the saturation of the instability. This saturation is achieved by a decrease of $\varepsilon$ through the coefficient $\xi$ in equation (6.3a). As soon as the amplitude $\varepsilon$ gets smaller than the critical value for the onset of instability, the amplitudes of the free Kelvin modes vanish quickly. It leads to a slow decrease of $A_{0}$ and consequently to an increase of $\varepsilon$. Then the instability can grow up again. This phenomenon being repetitive, amplitude oscillations are observed in time. Our experiments have clearly shown that the amplitude oscillations are due to the geostrophic term in equation $(6.3 a)$, which is delayed compared to the amplitudes of the free Kelvin modes.

Figure $12(b)$ shows three experimental measurements of $\left|A_{1}\right|$ at the same Reynolds numbers as in figure 12(a). We find again the three different regimes depending on the Reynolds number : stable, unstable and stationary, unstable and intermittent. The transition from stable to unstable flow is very well predicted theoretically, since it corresponds to the linear threshold of the instability : it was plotted on the stability diagram of Fig. 8 in the last section. The transition from an unstable stationary flow to an unstable intermittent flow is also in excellent agreement with the predictions. Indeed, this transition has been plotted in Fig. 8 as a dashed line. It clearly separates the stationary experiments $(\square)$ from the intermittent experiments $(\boldsymbol{\Delta})$. This agreement validates the nonlinear system (6.3).

However, the theory does not predict very well the quantitative value of the fixed point $A_{1 f}$ in the stationary case : the experimental value is twice larger than the theoretical one. In a similar way, in the intermittent case, the amplitude of the oscillations are not very well predicted by the theory. Nevertheless, the theoretical period of these oscillations $t_{\mathrm{th}}=250$ matches well the experimental one $t_{\exp }=290$. 


\subsection{Fixed point}

The system (6.3) admits two fixed points. The first one is trivial and corresponds to $A_{1 f}=A_{2 f}=A_{0 f}=0$ and $\varepsilon_{f}$ given by equation (3.12) with $a_{i}=\infty$. The second one is obtained by looking for a solution of the system (6.3) which satisfies $\partial \varepsilon / \partial t=$ $\partial\left|A_{j}\right| / \partial t=\partial A_{0} / \partial t=0$. This solution can be obtained analytically and its complete calculation is reported in Appendix C. In this section we only give asymptotical results by considering that viscous and detuning effects or geostrophic and nonlinear effects are dominant for the saturation of the instability.

Just above the threshold, the viscous and the detuning effects are stronger than geostrophic and nonlinear effects for the damping of the instability. Under this assumption, the terms $\xi_{j}$ and $\sigma_{j}$ can be neglected in equations $(6.3 b)$ and $(6.3 c)$. However, the amplitude of the forced Kelvin mode $\varepsilon$ is still affected by the geostrophic and nonlinear term $\xi \varepsilon A_{0}$. Then, a simple expression for the fixed point $\varepsilon_{f}$ can be obtained

$$
\varepsilon_{f}=\left[\frac{\alpha_{1}^{\mathrm{r}} \alpha_{2}^{\mathrm{r}}}{n_{1} n_{2}}\left(1+\frac{\left(\alpha_{1}^{\mathrm{i}}-\alpha_{2}^{\mathrm{i}}\right)^{2}}{\left(\alpha_{1}^{\mathrm{r}}+\alpha_{2}^{\mathrm{r}}\right)^{2}}\right)\right]^{1 / 2} .
$$

We recall that $\alpha_{j}^{\mathrm{r}}$ and $\alpha_{j}^{\mathrm{i}}$ are respectively the real and imaginary parts of the linear saturating term $\alpha_{j}$ given in equation (4.14).

As for the instability threshold, the linear saturation term can be either volume viscous effects $\left(R e \ll R e_{1}\right)$, surface viscous effects $\left(R e_{1} \ll R e \ll R e_{2}\right)$ or detuning effects $(R e \gg$ $\left.R e_{2}\right)$. This leads to various scalings for the amplitude of the forced Kelvin mode $\varepsilon_{\mathrm{f}}$ at the fixed point. These scalings are indicated on Fig. 4.

Far from the threshold, the geostrophic and the nonlinear effects are stronger than the viscous and the detuning effects. Under this assumption, the terms $\alpha_{j}$ can be neglected in equations $(6.3 b)$ and $(6.3 c)$, which leads to a simple expression for the fixed point

$$
\varepsilon_{f}=\left[\frac{\left(\alpha_{1}^{\mathrm{r}} \alpha_{2}^{\mathrm{r}}\right)^{1 / 2}}{\left|\alpha_{1}^{\mathrm{r}}+\alpha_{2}^{\mathrm{r}}\right|}\left|\frac{R o f}{\left(n_{1} n_{2}\right)^{1 / 2}}\right|\left|\frac{\widetilde{\xi}_{1}-\widetilde{\xi}_{2}}{\xi}\right|\right]^{1 / 2} .
$$

The definition of the parameters $\widetilde{\xi}_{j}$ is given in Appendix C.

A simple expression for the fixed point $A_{0 f}$ can also be obtained

$$
A_{0 f}=\left[\frac{\left|\left(\alpha_{1}^{\mathrm{r}}+\alpha_{2}^{\mathrm{r}}\right)\right|}{\left(\alpha_{1}^{\mathrm{r}} \alpha_{2}^{\mathrm{r}}\right)^{1 / 2}}\left|\frac{\operatorname{Rof}\left(n_{1} n_{2}\right)^{1 / 2}}{\xi\left(\widetilde{\xi}_{1}-\widetilde{\xi}_{2}\right)}\right|\right]^{1 / 2} .
$$

This fixed point does not depend on the Reynolds number : it is completely determined by the nonlinear terms. From equations (6.5) and (6.6) we observe that $\varepsilon_{f}$ and $A_{0 f}$ scale as $\sim R o^{1 / 2}$. The transition between the two regimes is shown schematically in figure 4 as a dashed line. It is simply found by equating (6.5) and (6.4) and leads again to three different scalings depending on the Reynolds number (see Fig. 4).

\subsection{Fixed point and mean flow}

In this section we compare the theoretical fixed point with the experimental results. The exact value of the fixed point $A_{1, \mathrm{f}}$ can be calculated by solving numerically the polynomial equation (D 10). It is plotted in Fig. 13( $a$ ) as a function of $R o$ for $R e=3664$ (thick line). We observe that the instability is weakly subcritical because the fixed point can exist for Rossby numbers slightly smaller than the linear threshold represented by the first vertical dash-dotted line. However, we were not able to assess the nature of the 


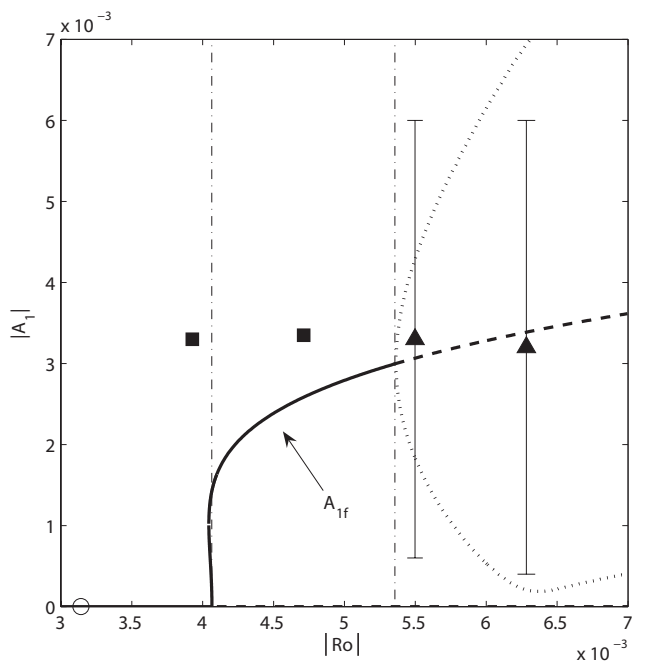

(a)

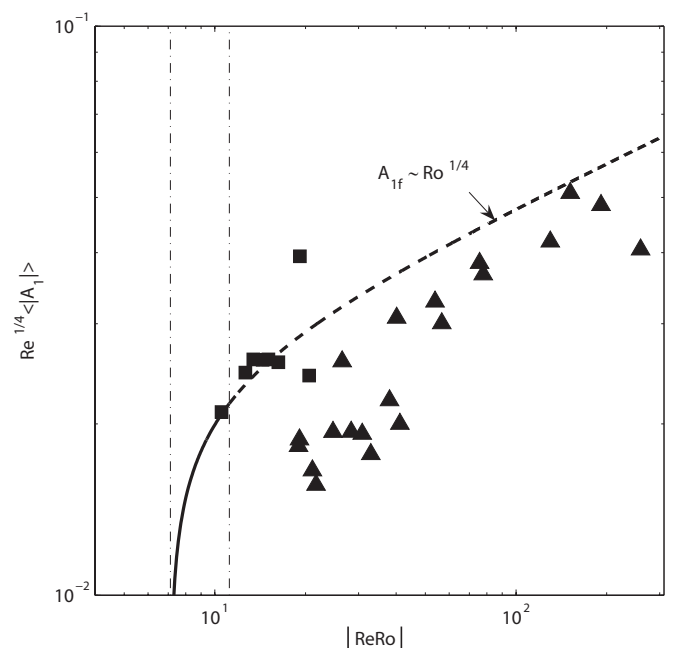

(b)

Figure 13. Amplitude of the free Kelvin mode $m_{1}=5$ as a function of $R o$ for $R e=3664$ (a) and as a function of RoRe without volume viscous effects (b). The fixed point calculated from the weakly nonlinear theory is represented by a solid line in the unstable and stationary regime and by a dashed line in the unstable and intermittent regime. Dotted lines represent the maximum and the minimum of oscillations of $A_{1}$. Dash-dotted lines represent the two thresholds. Squares $(\boldsymbol{\square})$ represent unstable and stationary experiments. Triangles $(\boldsymbol{\Delta})$ represent the mean value of $\left|A_{1}\right|$ in the unstable and intermittent regime. Amplitude ranges indicate the maximum and minimum values of $A_{1} . h=1.62, \omega=1.18$.

bifurcation experimentally because it would require to vary the precessing angle with an accuracy of $0.01^{\circ}$.

The second vertical dash-dotted line represents the transition from stationary to intermittent flow, i.e. the destabilization of the fixed point. The dashed line is the value of the unstable fixed point $A_{1, \mathrm{f}}$ and the two dotted branches represent the maximum and minimum values of $\left|A_{1}\right|$ obtained numerically, by integrating the nonlinear system. They show that the bifurcation is supercritical.

Experimental time-averaged values of $\left|A_{1}\right|$ are also shown on this graph as symbols. They are in rough agreement with the value of the fixed point. In the intermittent regime, the amplitude ranges indicate the maximum and minimum of the oscillations of $\left|A_{1}\right|$, which are of the same order of the theoretical predictions.

For high Reynolds numbers (i.e. $R e \gg R e_{1}$ ) volume viscous effects can be neglected compared to surface viscous effects. Under this assumption (and in the absence of detuning effects), it can be shown that $R e^{1 / 4} A_{1 f}$ only depends on $R e R o$ by rescaling the nonlinear equations. This is why we have plotted this quantity in Fig. $13(b)$ in order to collapse all the experimental results on a single curve. The theoretical value of the fixed point (shown as a line) increases and scales as $R o^{1 / 4}$ far from the threshold.

The mean amplitude $\left\langle\left|A_{1}\right|>\right.$ found experimentally is also plotted on the same graph and we observe that this mean amplitude is slightly overestimated by the fixed point $A_{1 f}$, especially at low ReRo. This may come from the effect of volume viscous terms which are neglected in this theory. However, the scaling for the mean flow is well predicted for high ReRo numbers. This means that the mean flow inside a precessing cylinder can be fairly well predicted even for very high Reynolds number (up to $R e \simeq 40,000$ ). It is surprising 


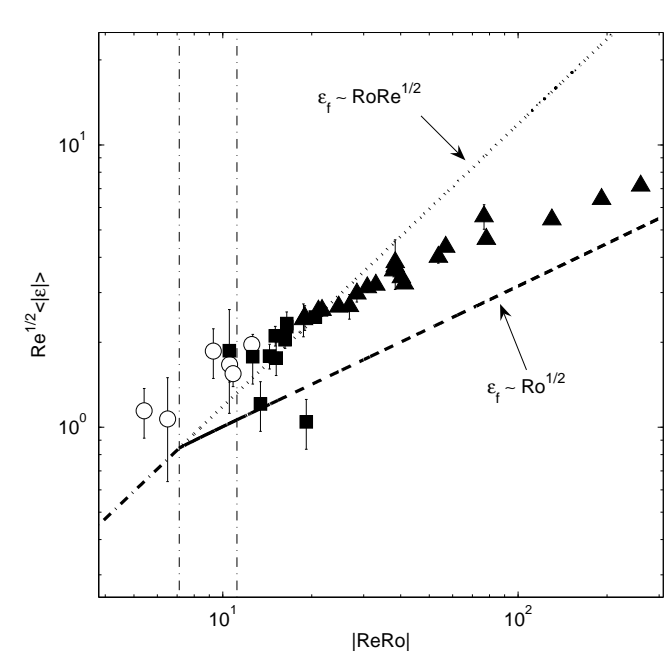

(a)

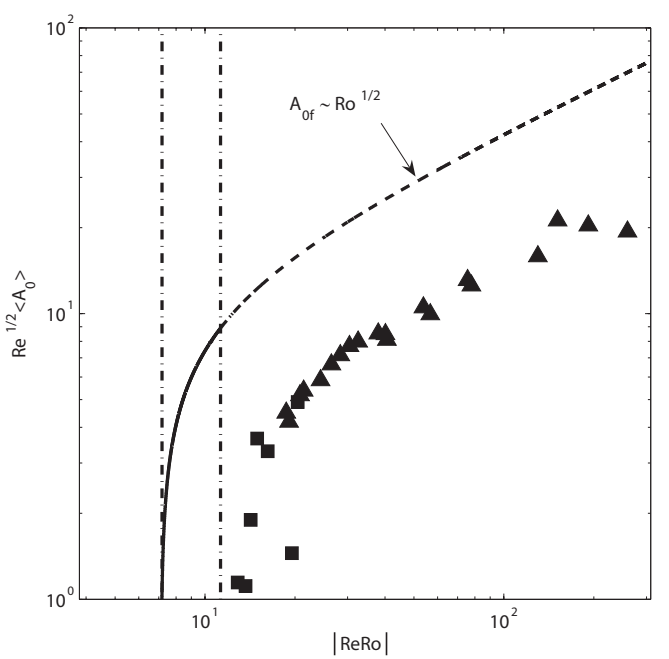

(b)

Figure 14. Amplitude of the forced Kelvin mode (a) and the geostrophic Kelvin mode (b) as a function of RoRe (volume viscous effects were neglected). The viscous saturating amplitude from equation (3.12) is represented by a dash-dotted line below the threshold (first vertical dash-dotted line) and a dotted line above the threshold. The fixed point (solid or dashed line) is compared to the experimental time averaged values $\langle|\varepsilon|\rangle(\mathrm{a})$ and $\left\langle A_{0}\right\rangle$ (b). These values are represented by circles (o) (stable flow), squares (ם) (unstable and stationary flow) or triangles ( $\mathbf{\Delta})$ (unstable and intermittent flow). $h=1.62, \omega=1.18$.

to see that this weakly nonlinear model is still efficient one decade above the threshold of the instability, in a regime where the flow is turbulent.

Figure 14(a) compares the fixed point $\varepsilon_{f}$ with the experimental mean amplitude $\langle|\varepsilon|\rangle$ of the forced Kelvin mode. As above, neglecting the volume viscous terms implies that $R e^{1 / 2} \varepsilon_{f}$ only depends on $R e R o$, which allow to collapse all the experimental results on a single curve. Below the threshold (first dash-dotted line, $|R e R o|<7.11$ ) the flow is stable such that $\varepsilon_{f}$ is equal to the viscously saturated amplitude (3.12), which is plotted as a thick dash-dotted line (extended by a dotted line above the threshold). Above the threshold the flow is unstable such that $\varepsilon$ tends to the fixed point $\varepsilon_{f}$ represented by a solid line. This fixed point becomes unstable above the second dash-dotted line $(|R e R o|=11.2)$, meaning that the flow becomes intermittent. The fixed point is then plotted as a dashed line and scales as $\varepsilon_{f} \sim R o^{1 / 2}$ (independently of the Reynolds number).

By comparing the fixed point and the experiments one can observe that even if the experimental values are underestimated at low $R e R o$, the discrepancy decreases at high ReRo. Moreover, the scaling for the mean flow is fairly well predicted. As explained previously this good agreement is surprising because the flow is turbulent at these high Reynolds numbers.

Finally, Fig. 14(b) compares the fixed point $A_{0 f}$ with the experimental mean amplitude $\left\langle A_{0}\right\rangle$ of the geostrophic Kelvin mode. Once again, neglecting the volume viscous terms allow to collapse all the results on a single curve $R e^{1 / 2} A_{0}$ as a function of $R e R o$. Below the threshold (first dash-dotted line, $|R e R o|<7.11$ ) the flow is stable such that $A_{0 f}=0$. Above the threshold the fixed point (solid line) increases and becomes unstable above the second dash-dotted line (dashed line). The fixed point scales as $A_{0 f} \sim R o^{1 / 2}$ at high Reynolds numbers. Even if the experimental values are strongly overestimated, the scaling for the mean flow is correctly predicted at high ReRo. 
We have shown in this section that a weakly nonlinear model is able to predict the properties of the instability at the threshold but also the characteristics of the flow at high Reynolds number in a very turbulent regime.

\section{Conclusion}

In this paper the instability of a fluid inside a precessing cylinder has been theoretically studied and compared with experiments.

Precession forces a Kelvin mode which is resonant for particular frequencies and thus is dominant compared to the rest of the flow. A linear stability analysis based on a mechanism of triadic resonance between Kelvin modes has been carried out and allows to obtain an analytical expression for the instability growth rate and threshold. We have shown that it is damped by volume viscous effects at low Reynolds numbers and by surface viscous effects due to Ekman layers at high Reynolds numbers. We have also shown that when a Kelvin mode combination is not exactly resonant the growth rate of the instability is also damped by detuning effects.

The structure of the unstable Kelvin modes, the growth rate of the instability and the stability diagram are in excellent agreement with experimental results. We deduce from this theory the general properties of the precessing instability for any aspect ratio and precession frequency. Several bands of instabilities with different azimuthal wavenumbers are predicted, which are confirmed experimentally. The instability always occur close to a resonance of a forced Kelvin mode since the amplitude of the base flow is larger than far from a resonance.

A weakly nonlinear analysis has also been carried out by taking into account the interactions between the two free Kelvin modes. The instability is saturated by the presence of a geostrophic mode which lowers the amplitude of the forced Kelvin mode. This weakly nonlinear model allows to predict the nature of the bifurcation and the properties of the unstable saturated flow just above the threshold. This model also predicts correctly the presence of an intermittent flow at higher Reynolds number. Finally, it is surprising to see that this low order model gives correct estimates for the mean velocity inside the cylinder at very high Reynolds numbers even when the flow is turbulent.

The nonlinear model is essentially based on the fact that the nonlinear interaction of a Kelvin mode with itself in the Ekman layers leads to the presence of a geostrophic mode. However, this interaction vanishes in the inviscid case and viscous effects are needed in order to predict the generation of the geostrophic mode. In this paper, this term was adjusted to the experiments by a fitting parameter. A correct analysis of nonlinear and viscous interaction of Kelvin modes would thus be highly useful in order to get analytical expressions for the generation of geostrophic motion.

In the future, it would be interesting to see if this instability mechanism can be observed in an ellipsoid, as predicted theoretically by Kerswell (1993). If it is the case, we expect the scalings observed in Fig. 4 and Fig. 8 to be still valid although the quantitative values will have to be calculated in a different manner. This could highly improve the comprehension of the flow inside the outer core of the earth and thus shed light on the geodynamo that takes place there.

It would also be interesting to see how these Kelvin modes interact with a magnetic field if the liquid is conductor. Since the structure of the unstable flow is here predicted for any Reynolds number, a theoretical study of the growth of the magnetic field in such a flow is now possible. This could explain the results by Gans (1970a) who observed an unstable magnetic field in a precessing cylinder full of liquid sodium.

Finally, the destabilization of a fluid-filled gyroscope by precession has been extensively 
studied under the assumption that the flow inside the gyroscope is stable and laminar. These results can now be extended to the case of an unstable fluid cylinder owing to the model presented in this paper.

We would like to thank Wietze Herreman for very fruitful discussions, especially concerning the weakly nonlinear theory. This study was carried out under CEA-CNRS contract No. 012171.

\section{Annexe A. Base flow calculation}

The operators appearing in the complex Navier-Stokes equation (3.5) are defined by

$$
\begin{gathered}
\mathcal{I}=\left(\begin{array}{cccc}
1 & 0 & 0 & 0 \\
0 & 1 & 0 & 0 \\
0 & 0 & 1 & 0 \\
0 & 0 & 0 & 0
\end{array}\right) \\
\mathcal{D}=\left(\begin{array}{cccc}
0 & 0 & -\mathrm{i} & 0 \\
0 & 0 & 1 & 0 \\
\mathrm{i} & -1 & 0 & 0 \\
0 & 0 & 0 & 0
\end{array}\right) \\
\mathcal{L}=\left(\begin{array}{cccc}
\Delta-\frac{1}{r^{2}} & -\frac{2}{r^{2}} \frac{\partial}{\partial \varphi} & 0 & 0 \\
\frac{2}{r^{2}} \frac{\partial}{\partial \varphi} & \Delta-\frac{1}{r^{2}} & 0 & 0 \\
0 & 0 & \Delta & 0 \\
0 & 0 & 0 & 0
\end{array}\right)
\end{gathered}
$$

where

$$
\Delta=\frac{1}{r} \frac{\partial}{\partial r}+\frac{\partial^{2}}{\partial r^{2}}+\frac{1}{r^{2}} \frac{\partial^{2}}{\partial \varphi^{2}}+\frac{\partial^{2}}{\partial z^{2}}
$$

and

$$
\mathcal{M}=\left(\begin{array}{cccc}
0 & -2 & 0 & \frac{\partial}{\partial r} \\
2 & 0 & 0 & \frac{1}{r} \frac{\partial}{\partial \varphi} \\
0 & 0 & 0 & \frac{\partial}{\partial z} \\
\frac{\partial}{\partial r}+\frac{1}{r} & \frac{1}{r} \frac{\partial}{\partial \varphi} & \frac{\partial}{\partial z} & 0
\end{array}\right)
$$

The vectors $\mathbf{F}_{0}$ and $\mathbf{N}\left(\mathbf{v}_{1}, \mathbf{v}_{2}\right)$ are defined by

$$
\mathbf{F}_{0}=\left(\begin{array}{c}
0 \\
0 \\
-r \omega \\
0
\end{array}\right)
$$

and

$$
\mathbf{N}\left(\mathbf{v}_{1}, \mathbf{v}_{2}\right)=\left(\begin{array}{c}
\left(\mathbf{v}_{1}+\overline{\mathbf{v}_{1}}\right) \times\left(\nabla \times \mathbf{v}_{2}\right) \\
0
\end{array}\right) .
$$

The linear and inviscid amplitude $a_{i}$ of the Kelvin modes $\mathbf{v}_{1, \omega_{i}, k_{i}}(r)$ inside a precessing cylinder in the non-resonant case is

$$
a_{i}=\frac{\omega^{2}}{(\omega-2)\left(k_{i}^{2}+1\right) k_{i} J_{1}\left(\delta_{i}\right) \cos \left(k_{i} h / 2\right)} .
$$


The vector $\mathbf{u}_{m_{i}, \omega_{i}, k_{i}}(r)$ of a Kelvin mode appearing in equation (3.9) is defined by

$$
\begin{aligned}
\mathbf{u}_{m_{i}, \omega_{i}, k_{i}}(r) & =\left(\begin{array}{c}
U_{m_{i}, \omega_{i}, k_{i}}(r) \\
V_{m_{i}, \omega_{i}, k_{i}}(r) \\
W_{m_{i}, \omega_{i}, k_{i}}(r) \\
P_{m_{i}, \omega_{i}, k_{i}}(r)
\end{array}\right) \\
= & \left(\begin{array}{c}
\frac{-1}{4-\omega_{i}^{2}}\left(\omega_{i} \delta_{i} J_{m_{i}}^{\prime}\left(\delta_{i} r\right)+2 \frac{m_{i}}{r} J_{m_{i}}\left(\delta_{i} r\right)\right) \\
\frac{-\mathrm{i}}{4-\omega_{i}^{2}}\left(2 \delta_{i} J_{m_{i}}^{\prime}\left(\delta_{i} r\right)+\frac{\omega_{i} m_{i}}{r} J_{m_{i}}\left(\delta_{i} r\right)\right) \\
\mathrm{i} \frac{k_{i}}{\omega_{i}} J_{m_{i}}\left(\delta_{i} r\right) \\
-\mathrm{i} J_{m_{i}}\left(\delta_{i} r\right)
\end{array}\right) .
\end{aligned}
$$

We introduce the scalar product

$$
\mathbf{X} \odot \mathbf{Y}=\int_{V}\left(\overline{X_{r}} Y_{r}+\overline{X_{\varphi}} Y_{\varphi}+\overline{X_{z}} Y_{z}+\overline{X_{p}} Y_{p}\right) d^{3} V
$$

The forcing term $f$ appearing in equation (3.12) is defined by

$$
f=\frac{1}{\mathrm{i}} \frac{\mathbf{v}_{1, \omega, k} \odot \mathbf{F}_{0} e^{\mathrm{i}(\omega t+\varphi)}}{\mathbf{v}_{1, \omega, k} \odot \mathcal{I} \mathbf{v}_{1, \omega, k}} .
$$

For a resonant Kelvin mode, a simple expression of $f$ is

$$
f=\frac{(\omega+2) \omega\left(4-\omega^{2}\right)^{2}}{\delta^{2} h J_{1}(\delta)\left[\omega^{2}\left(\omega+2 \delta^{2}-2\right)-4 \omega+8\right]} .
$$

\section{Annexe B. Linear stability analysis coefficients}

\section{B.1. Nonlinear coefficients}

The nonlinear coefficients $n_{1}$ and $n_{2}$ appearing in equation (4.13) are defined by

$$
\begin{aligned}
& n_{1}=\frac{\mathbf{v}_{1} \odot\left[\mathbf{N}\left(\mathbf{v}_{1, \omega, k}, \mathbf{v}_{2}\right)+\mathbf{N}\left(\mathbf{v}_{2}, \overline{\mathbf{v}}_{1, \omega, k}\right)\right]}{\mathbf{v}_{1} \odot \mathcal{I} \mathbf{v}_{1}}, \\
& n_{2}=\frac{\mathbf{v}_{2} \odot\left[\mathbf{N}\left(\mathbf{v}_{1, \omega, k}, \mathbf{v}_{1}\right)+\mathbf{N}\left(\mathbf{v}_{1}, \mathbf{v}_{1, \omega, k}\right)\right]}{\mathbf{v}_{2} \odot \mathcal{I} \mathbf{v}_{2}}
\end{aligned}
$$

where the vectors $\mathbf{v}_{1, \omega, k}$ and $\mathbf{v}_{j}$ are respectively given by equations (3.9) and (4.7). Note that the definition of $n_{j}$ does not depend on the choice of the sign plus or minus in equation (4.7).

By using the formula $\nabla \times \mathbf{v}_{K}=\frac{2}{\mathrm{i} \omega} \frac{\partial}{\partial z} \mathbf{v}_{K}$ for a Kelvin mode $\mathbf{v}_{K}$, a development of (B 1a) and (B 1b) can be given as

$$
n_{1}=-8 \pi h \frac{ \pm \frac{k_{2}}{\omega_{2}}-\frac{k}{\omega}}{\mathbf{v}_{1} \odot \mathcal{I} \mathbf{v}_{1}} \int_{0}^{1}\left|\begin{array}{ccc}
U_{m, \omega, \pm k} & U_{m_{1}, \omega_{1}, k_{1}} & \bar{U}_{m_{2}, \omega_{2}, k_{2}} \\
V_{m, \omega, \pm k} & V_{m_{1}, \omega_{1}, k_{1}} & \bar{V}_{m_{2}, \omega_{2}, k_{2}} \\
W_{m, \omega, \pm k} & W_{m_{1}, \omega_{1}, k_{1}} & \bar{W}_{m_{2}, \omega_{2}, k_{2}}
\end{array}\right| r d r
$$

and

$$
n_{2}=8 \pi h \frac{ \pm \frac{k_{1}}{\omega_{1}}-\frac{k}{\omega}}{\mathbf{v}_{2} \odot \mathcal{I} \mathbf{v}_{2}} \int_{0}^{1}\left|\begin{array}{ccc}
U_{m, \omega, \pm k} & U_{m_{1}, \omega_{1}, k_{1}} & \bar{U}_{m_{2}, \omega_{2}, k_{2}} \\
V_{m, \omega, \pm k} & V_{m_{1}, \omega_{1}, k_{1}} & \bar{V}_{m_{2}, \omega_{2}, k_{2}} \\
W_{m, \omega, \pm k} & W_{m_{1}, \omega_{1}, k_{1}} & \bar{W}_{m_{2}, \omega_{2}, k_{2}}
\end{array}\right| r d r
$$

where the operator $|$.$| is the determinant.$

The plus sign corresponds to the case $k_{2}-k_{1}=k$. 
The minus sign corresponds to the case $k_{1}-k_{2}=k$.

We recall that equations (B 2) and (B 3) are only valid for free Kelvin modes with different parities with respect to $z$, i.e. at (or close to) a resonance of the forced Kelvin mode.

\section{B.2. Viscous coefficients}

The boundary viscous coefficient $s_{j}$ appearing in equation (4.13) is defined by

$$
\frac{s_{j}}{R e^{1 / 2}}=\frac{\mathbf{v}_{j} \odot\left(\frac{\partial}{\partial t} \mathcal{I}+\mathcal{M}\right) \mathbf{v}_{j}^{(1)}}{\mathbf{v}_{j} \odot \mathcal{I} \mathbf{v}_{j}}
$$

where $\mathbf{v}_{j}^{(1)}$ is the vector appearing in equation (4.12). Integrating by part, we can show that

$$
\begin{aligned}
\mathbf{v}_{j} \odot\left(\frac{\partial}{\partial t} \mathcal{I}+\mathcal{M}\right) \mathbf{v}_{j}^{(1)} & =-\overline{\mathbf{v}_{j}^{(1)} \odot\left(\frac{\partial}{\partial t} \mathcal{I}+\mathcal{M}\right) \mathbf{v}_{j}} \\
& +\int_{\text {surface }}\left(\overline{\mathbf{v}}_{j}^{\perp} \cdot \mathbf{v}_{j}^{(1) p}+\overline{\mathbf{v}}_{j}^{p} \cdot \mathbf{v}_{j}^{(1) \perp}\right) d S
\end{aligned}
$$

where $\perp$ stands for the perpendicular component to the wall and the subscript $p$ stands for the pressure component. The first right-hand side term of this last equation equals 0 because $\mathbf{v}_{j}$ is in the kernel of the operator $\left(\frac{\partial}{\partial t} \mathcal{I}+\mathcal{M}\right)$. The first term in the surface integral is also equal to 0 because $\mathbf{v}_{j}^{\perp}$ vanishes at the walls since it is a free Kelvin mode. The second term inside the integral can be calculated because $\mathbf{v}_{j}^{(1) \perp}$ is given by the no-slip boundary condition at order $\varepsilon$, which means that it is the opposite of the Ekman pumping (of order $R e^{-1 / 2} \sim \varepsilon$ ) created by the Kelvin modes $\mathbf{v}_{j}$ of order one. The determination of the velocity in the boundary layer is classical. The reader could refer to Greenspan (1968) or for the peculiar case of Kelvin modes to Kudlick (1966). The viscous coefficients can thus be calculated as

$$
s_{j}=2 \frac{I_{j}^{\prime} r+I_{j}^{\prime z}}{\mathbf{v}_{j} \odot \mathcal{I} \mathbf{v}_{j}},
$$

where

and

$$
I_{j}^{\prime r}=2 \pi h \bar{P}_{m_{j}, \omega_{j}, k_{j}}(1)\left[\frac{-\mathrm{i}}{K_{j}}\left(m_{j} V_{m_{j}, \omega_{j}, k_{j}}(1)+k_{j} W_{m_{j}, \omega_{j}, k_{j}}(1)\right)\right],
$$

$$
I_{j}^{\prime z}=\frac{\sqrt{2} \pi(1-\mathrm{i})}{\omega_{j}^{2}}\left(\frac{J_{m_{j}}^{2}\left(\delta_{j}\right)}{\left(2-\omega_{j}\right)^{3 / 2}}+\frac{\mathrm{i} J_{m_{j}}^{2}\left(\delta_{j}\right)}{\left(2+\omega_{j}\right)^{3 / 2}}\right)\left[\left(\delta_{j}^{2}-m_{j}^{2}\right) \omega_{j}^{2}+4 m_{j}^{2}\right],
$$

with

$$
K_{j}=\left(\frac{\left|\omega_{j}\right|}{2}\right)^{1 / 2}\left(1+\mathrm{i} \operatorname{sgn}\left(\omega_{j}\right)\right) .
$$

The same boundary viscous effects appear on the forced Kelvin mode $\mathbf{v}_{\mathbf{1}, \omega, \mathbf{k}}$ and can be calculated in the same manner. If we note $\widetilde{\mathbf{v}}_{1, \omega, k}$ the Ekman pumping created by the Kelvin mode $\mathbf{v}_{1, \omega, k}$, the boundary viscous coefficient $s$ appearing in equation (3.12) is given by

$$
\frac{s}{R e^{1 / 2}}=\frac{\mathbf{v}_{1, \omega, k} \odot\left(\frac{\partial}{\partial t} \mathcal{I}+\mathcal{M}\right) \widetilde{\mathbf{v}}_{1, \omega, k}}{\mathbf{v}_{1, \omega, k} \odot \mathcal{I} \mathbf{v}_{1, \omega, k}}=\frac{2}{R e^{1 / 2}} \frac{I^{\prime r}+I^{\prime z}}{\mathbf{v}_{1, \omega, k} \odot \mathcal{I} \mathbf{v}_{1, \omega, k}},
$$


where $I^{\prime r}$ and $I^{\prime} z$ are respectively given by $I_{j}^{\prime r}$ and $I_{j}^{\prime} z$ with $m_{j}=1, \omega_{j}=\omega$ and $k_{j}=k$.

The volume viscous coefficient $v_{j}$ appearing in equation (4.13) is defined by

$$
v_{j}=-\frac{\mathbf{v}_{j} \odot \mathcal{L} \mathbf{v}_{j}}{\mathbf{v}_{j} \odot \mathcal{I} \mathbf{v}_{j}}=k_{j}^{2}+\delta_{j}^{2} .
$$

\section{B.3. Detuning coefficients}

The detuning coefficient $q_{j}$ appearing in equation (4.13) is defined by

$$
q_{j}=\frac{\mathbf{v}_{j} \odot \mathcal{M}_{\Delta} \mathbf{v}_{j}}{\mathbf{v}_{j} \odot \mathcal{I} \mathbf{v}_{j}},
$$

with

$$
\mathcal{M}_{\Delta}=\frac{1}{\mathrm{i} k_{j}}\left(\begin{array}{cccc}
0 & 0 & 0 & 0 \\
0 & 0 & 0 & 0 \\
0 & 0 & 0 & \frac{\partial}{\partial z} \\
0 & 0 & \frac{\partial}{\partial z} & 0
\end{array}\right)
$$

The calculation gives

$$
q_{j}=-8 \pi h \frac{k_{j}}{\omega_{j}} \int_{0}^{1} J_{m_{j}}^{2}\left(\delta_{j} r\right) r d r / \mathbf{v}_{j} \odot \mathcal{I} \mathbf{v}_{j}
$$

\section{Annexe C. Nonlinear calculation}

The aim of this Appendix is to calculate the coefficients $\sigma_{j}, \xi_{j}$ and $\lambda$ appearing in the weakly nonlinear amplitude equations (6.3).

\section{C.1. Coefficients $\sigma_{j}$}

The nonlinear interaction of a Kelvin mode $\mathbf{v}_{j}$ with itself gives rise to a flow $\mathbf{v}_{2_{j}}$ such that

$$
\left(\frac{\partial}{\partial t} \mathcal{I}+\mathcal{M}\right) \mathbf{v}_{2_{j}}+\text { c.c. }=\mathbf{N}\left(\mathbf{v}_{j}, \mathbf{v}_{j}\right)+\text { c.c. }
$$

As shown by Waleffe (1989), $\mathbf{v}_{2_{j}}$ has two velocity components because $\mathbf{v}_{j}$ is the sum of two waves with opposite axial wavenumbers $+k_{j}$ and $-k_{j}$. For a Kelvin mode $\left(m_{j}, \omega_{j}, k_{j}\right)$ these two components are of the form $\left(2 m_{j}, 2 k_{j}, 0\right)$ and $\left(0,0,2 k_{j}\right)$ and correspond to the two vectors

$$
\mathbf{v}_{2 k_{j}}= \pm\left(\begin{array}{c}
0 \\
a_{2 k_{j}} \cos \left(2 k_{j} z\right) \\
0 \\
p_{2 k_{j}} \cos \left(2 k_{j} z\right)
\end{array}\right), \mathbf{v}_{2 \omega_{j}}= \pm\left(\begin{array}{c}
\frac{\mathrm{i} m_{j}}{r} a_{2 \omega_{j}} \\
-\frac{1}{2} \frac{d a_{2 \omega_{j}}}{d r} \\
0 \\
p_{2 \omega_{j}}
\end{array}\right)
$$

with

and

$$
a_{2 k_{j}}=4 \frac{\mathrm{i} k_{j}}{\omega_{j}}\left(\mathrm{i} V_{m_{j}, \omega_{j}, k_{j}} W_{m_{j}, \omega_{j}, k_{j}}+\frac{1}{2 k_{j}} \frac{d}{d r}\left(U_{m_{j}, \omega_{j}, k_{j}} V_{m_{j}, \omega_{j}, k_{j}}\right)\right),
$$

$$
a_{2 \omega_{j}}=4 \frac{\delta_{j}^{2}}{\left(\omega_{j}^{2}-4\right)^{2}}\left[J_{m_{j}}^{2}\left(\delta_{j} r\right)-J_{m_{j}}^{2}\left(\delta_{j}\right) r^{2 m_{j}}\right] .
$$


For a free Kelvin mode with a minus (resp. plus) sign in its definition (4.7) the plus (resp. minus) sign must be chosen in equation (C 2). The coefficient $\sigma_{j}$ in (6.3) is separated into two parts. The first one comes from the nonlinear interaction of $\mathbf{v}_{j}$ and $\mathbf{v}_{2 k_{j}}$

$$
\sigma_{2 k_{j}}=\mathbf{v}_{j} \odot\left[\mathbf{N}\left(\mathbf{v}_{2 k_{j}}, \mathbf{v}_{j}\right)+\mathbf{N}\left(\mathbf{v}_{j}, \mathbf{v}_{2 k_{j}}+\text { c.c. }\right)\right] .
$$

The calculation gives

$$
\sigma_{2 k_{j}}=8 \pi h \int_{0}^{1}\left(2 \mathrm{i} k_{j} a_{2 k_{j}} V_{m_{j}, \omega_{j}, k_{j}} W_{m_{j}, \omega_{j}, k_{j}}+a_{2 k_{j}} \frac{d\left(U_{m_{j}, \omega_{j}, k_{j}} V_{m_{j}, \omega_{j}, k_{j}}\right)}{d r}\right) r d r,
$$

with $a_{2 k_{j}}$ given by equation (C 3 ).

The other term comes from the interaction of $\mathbf{v}_{j}$ with $\mathbf{v}_{2 \omega_{j}}$

$$
\sigma_{2 \omega_{j}}=\mathbf{v}_{j} \odot \mathbf{N}\left(\mathbf{v}_{2 \omega_{j}} \mathrm{e}^{2 \mathrm{i}\left(\omega_{j} t+m_{j} \varphi\right)}, \overline{\mathbf{v}_{j}}\right) .
$$

The calculation gives

$$
\sigma_{2 \omega_{j}}=8 \pi h \frac{\mathrm{i} k_{j}}{\omega_{j}} \int_{0}^{1}\left(\frac{d a_{2 \omega_{j}}}{d r} \mathrm{i} U_{m_{j}, \omega_{j}, k_{j}} W_{m_{j}, \omega_{j}, k_{j}}+\frac{2 m_{j} a_{2 \omega_{j}}}{r} V_{m_{j}, \omega_{j}, k_{j}} W_{m_{j}, \omega_{j}, k_{j}}\right) r d r
$$

The final coefficient $\sigma_{j}$ used in the weakly nonlinear amplitude equations (6.3) is

$$
\sigma_{j}=-\frac{\sigma_{2 k_{j}}+\sigma_{2 \omega_{j}}}{\mathrm{i} \mathbf{v}_{j} \odot \mathcal{I} \mathbf{v}_{j}}
$$

\section{C.2. Coefficients $\xi_{j}$}

The coefficients $\xi_{j}$ of (6.3) describe the nonlinear interaction of the $j$-th free Kelvin mode with the geostrophic mode

$$
\xi_{j}=\mathrm{i} \mathbf{v}_{j} \odot\left[\mathbf{N}\left(\frac{1}{2} \mathbf{v}_{0}, \mathbf{v}_{j}\right)+\mathbf{N}\left(\mathbf{v}_{j}, \mathbf{v}_{0}\right)\right] / \mathbf{v}_{\mathbf{j}} \odot \mathcal{I} \mathbf{v}_{\mathbf{j}} .
$$

We then obtain

$$
\xi_{j}=8 \mathrm{i} \pi h \frac{\int_{0}^{1}\left(\frac{2 k_{j}}{\omega_{j}} v_{0} U_{m_{j}, \omega_{j}, k_{j}} W_{m_{j}, \omega_{j}, k_{j}}+\frac{1}{r} \frac{d}{d r}\left(r v_{0}\right) U_{m_{j}, \omega_{j}, k_{j}} V_{m_{j}, \omega_{j}, k_{j}}\right) r d r}{\mathbf{v}_{j} \odot \mathcal{I} \mathbf{v}_{j}},
$$

where $v_{0}$ is the orthoradial component of the geostrophic flow given by (6.1). For the forced Kelvin mode, $\mathbf{v}_{j} \odot \mathcal{I} \mathbf{v}_{j}$ is replaced by $\mathbf{v}_{1, \omega, k} \odot \mathcal{I} \mathbf{v}_{1, \omega, k}$ in (C 11) in order to obtain the coefficient $\xi$.

\section{C.3. Coefficient $\lambda$}

The parameter $\lambda$ describes the nonlinear interaction between the first and the second free Kelvin mode. This parameter appears in equation (6.3a) and is given by

$$
\lambda=\frac{\mathbf{v}_{1, \omega, k} \odot\left[\mathbf{N}\left(\mathbf{v}_{1}, \mathbf{v}_{2}\right)+\mathbf{N}\left(\mathbf{v}_{2}, \overline{\mathbf{v}}_{1}\right)\right]}{\mathbf{v}_{1, \omega, k} \odot \mathcal{I} \mathbf{v}_{1, \omega, k}} .
$$

We obtain 


$$
\lambda= \pm 8 \pi h \frac{\frac{k_{2}}{\omega_{2}}-\frac{k_{1}}{\omega_{1}}}{\mathbf{v}_{1, \omega, k} \odot \mathcal{I} \mathbf{v}_{1, \omega, k}} \int_{0}^{1}\left|\begin{array}{ccc}
U_{m, \omega, \pm k} & U_{m_{1}, \omega_{1}, k_{1}} & \bar{U}_{m_{2}, \omega_{2}, k_{2}} \\
V_{m, \omega, \pm k} & V_{m_{1}, \omega_{1}, k_{1}} & \bar{V}_{m_{2}, \omega_{2}, k_{2}} \\
W_{m, \omega, \pm k} & W_{m_{1}, \omega_{1}, k_{1}} & W_{m_{2}, \omega_{2}, k_{2}}
\end{array}\right| r d r .
$$

The plus sign corresponds to the case $k_{2}-k_{1}=k$.

The minus sign corresponds to the case $k_{1}-k_{2}=k$.

\section{Annexe D. Fixed point}

To obtain a fixed point $\left(\varepsilon_{f}, A_{1 f}, A_{2 f}, A_{0 f}\right)$ of the nonlinear amplitude equations (6.3), we search a solution $\left(\varepsilon, A_{1}, A_{2}, A_{0}\right)$ such that $\frac{\partial \varepsilon}{\partial t}=\frac{\partial A_{0}}{\partial t}=0$ and $A_{j}=A_{j}^{0} \mathrm{e}^{\mathrm{i} \tilde{\omega} t}$ with $\frac{\partial A_{j}^{0}}{\partial t}=0$ and $\tilde{\omega}$ real. Then, the fixed point satisfies the following system

$$
\begin{aligned}
\varepsilon\left(\alpha+\mathrm{i} \xi A_{0}\right) & =\mathrm{i} f R o, \\
\alpha_{1}+\mathrm{i}\left(\widetilde{\omega}+\xi_{1} A_{0}+\sigma_{1}\left|A_{1}^{0}\right|^{2}\right) & =\bar{\varepsilon} n_{1} \frac{A_{2}^{0}}{A_{1}^{0}}, \\
\alpha_{2}+\mathrm{i}\left(\widetilde{\omega}+\xi_{2} A_{0}+\sigma_{2}\left|A_{2}^{0}\right|^{2}\right) & =\varepsilon n_{2} \frac{A_{1}^{0}}{A_{2}^{0}}, \\
\frac{h}{2}\left(\chi_{1}\left|A_{1}^{0}\right|^{2}+\chi_{2}\left|A_{2}^{0}\right|^{2}\right) & =A_{0} .
\end{aligned}
$$

Here, we have neglected the term $\lambda \overline{A_{1}} A_{2}$ because it was very small in our case, but it can be easily added and the system can be solved in the same manner. The product of $(\mathrm{D} 1 b)$ by $(\mathrm{D} 1 c)$ is real such that the modulus of (D 1b) over (D 1c) equals the modulus of the real part of (D 1b) over the real part of (D 1c). Thus it comes

$$
\left|\frac{A_{1}^{0}}{A_{2}^{0}}\right|=\left|\frac{n_{2} \alpha_{1}^{\mathrm{r}}}{n_{1} \alpha_{2}^{\mathrm{r}}}\right|^{1 / 2} \text {. }
$$

Equation (D $1 d$ ) allows to obtain $\left|A_{1}^{0}\right|$ and $\left|A_{2 f}^{0}\right|$ as functions of $A_{0}$

$$
\begin{aligned}
& \left|A_{1}^{0}\right|=A_{1 f}=\left[\frac{2\left|n_{1} \alpha_{2}^{\mathrm{r}}\right|}{h\left(\chi_{1}\left|n_{1} \alpha_{2}^{\mathrm{r}}\right|+\chi_{2}\left|n_{2} \alpha_{1}^{\mathrm{r}}\right|\right)} A_{0}\right]^{1 / 2}, \\
& \left|A_{2}^{0}\right|=A_{2 f}=\left[\frac{2\left|n_{2} \alpha_{1}^{\mathrm{r}}\right|}{h\left(\chi_{1}\left|n_{1} \alpha_{2}^{\mathrm{r}}\right|+\chi_{2}\left|n_{2} \alpha_{1}^{\mathrm{r}}\right|\right)} A_{0}\right]^{1 / 2} .
\end{aligned}
$$

These two equations allow to replace $\sigma_{1}\left|A_{1}^{0}\right|^{2}$ and $\sigma_{2}\left|A_{2}^{0}\right|^{2}$ in equations (D $\left.1 b\right)$ and (D $1 c$ ) as a function of $A_{0}$. Thus (D 1$)$ rewrites

$$
\begin{aligned}
\varepsilon\left(\alpha+\mathrm{i} \xi A_{0}\right) & =\mathrm{i} f R o, \\
\alpha_{1}+\mathrm{i}\left(\widetilde{\omega}+\widetilde{\xi}_{1} A_{0}\right) & =\bar{\varepsilon} n_{1} \frac{A_{2}^{0}}{A_{1}^{0},} \\
\alpha_{2}+\mathrm{i}\left(\widetilde{\omega}+\widetilde{\xi}_{2} A_{0}\right) & =\varepsilon n_{2} \frac{A_{1}^{0}}{A_{2}^{0}}, \\
\frac{h}{2}\left(\chi_{1}\left|A_{1}^{0}\right|^{2}+\chi_{2}\left|A_{2}^{0}\right|^{2}\right) & =A_{0},
\end{aligned}
$$


with

and

$$
\widetilde{\xi}_{1}=\xi_{1}+\frac{2 \sigma_{1}\left|n_{1} \alpha_{2}^{\mathrm{r}}\right|}{h\left(\chi_{1}\left|n_{1} \alpha_{2}^{\mathrm{r}}\right|+\chi_{2}\left|n_{2} \alpha_{1}^{\mathrm{r}}\right|\right)},
$$

$$
\widetilde{\xi}_{2}=\xi_{2}+\frac{2 \sigma_{2}\left|n_{2} \alpha_{1}^{\mathrm{r}}\right|}{h\left(\chi_{1}\left|n_{1} \alpha_{2}^{\mathrm{r}}\right|+\chi_{2}\left|n_{2} \alpha_{1}^{\mathrm{r}}\right|\right)} .
$$

The product of equations (D $5 b)$ and (D $5 c$ ) gives a complex equation, whose imaginary part gives the nonlinear frequency of the free Kelvin modes

$$
\widetilde{\omega}=-\frac{\operatorname{Im}\left\{\left(\alpha_{1}+\mathrm{i} \widetilde{\xi}_{1} A_{0}\right)\left(\alpha_{2}+\mathrm{i} \widetilde{\xi}_{2} A_{0}\right)\right\}}{\alpha_{1}^{\mathrm{r}}+\alpha_{2}^{\mathrm{r}}},
$$

and whose real part thus leads to

$$
\begin{aligned}
|\varepsilon| & =\varepsilon_{f} \\
& =\left\{\frac{\alpha_{1}^{\mathrm{r}} \alpha_{2}^{\mathrm{r}}}{n_{1} n_{2}\left(\alpha_{1}^{\mathrm{r}}+\alpha_{2}^{\mathrm{r}}\right)^{2}}\left\{\left(\alpha_{1}^{\mathrm{r}}+\alpha_{2}^{\mathrm{r}}\right)^{2}+\left[\left(\alpha_{1}^{\mathrm{i}}-\alpha_{2}^{\mathrm{i}}\right)+\left(\widetilde{\xi}_{1}-\widetilde{\xi}_{2}\right) A_{0}\right]^{2}\right\}\right\}^{1 / 2} .
\end{aligned}
$$

By equating (D 9) and the modulus of (D $5 a)$ divided by $\left(\alpha+\mathrm{i} \xi A_{0}\right)$ we obtain an equation of order 4 for $A_{0}$

$$
\begin{array}{r}
\frac{\alpha_{1}^{\mathrm{r}} \alpha_{2}^{\mathrm{r}}\left[\alpha^{\mathrm{r} 2}+\left(\xi A_{0}+\alpha^{\mathrm{i}}\right)^{2}\right]}{\left(\alpha_{1}^{\mathrm{r}}+\alpha_{2}^{\mathrm{r}}\right)^{2}}\left\{\left(\alpha_{1}^{\mathrm{r}}+\alpha_{2}^{\mathrm{r}}\right)^{2}+\left[\left(\alpha_{1}^{\mathrm{i}}-\alpha_{2}^{\mathrm{i}}\right)+\left(\widetilde{\xi}_{1}-\widetilde{\xi}_{2}\right) A_{0}\right]^{2}\right\} \\
-R o^{2} f^{2} n_{1} n_{2}=0 .
\end{array}
$$

This equation has a unique real and positive solution which is $A_{0 f}$, except in the subcritical regime, where it can have two positive roots. Note that if we take into account the term $\lambda$ in equation $(6.3 a)$, the equation for $A_{0}$ becomes an equation of order 6 instead of 4 . Once $A_{0 f}$ is known, $\varepsilon_{f}$ can be determined via equation (D 9$)$ and $A_{1 f}$ and $A_{2 f}$ by equations (D 3) and (D 4).

\section{REFERENCES}

Agrawal, B. N. 1993 Dynamics characteristics of liquid motion in partially filled tanks of a spinning spacecraft. J. Guid. Control Dynam. 16 (4), 636-640.

Bao, G. W. \& Pascal, M. 1997 Stability of a spinning liquid filled spacecraft. Appl. Mech. 67, 407-421.

Bayly, B. J. 1986 Three-dimensional instability of elliptical flow. Phys. Rev. Lett. 57, 21602163.

Busse, F. 1968 Steady fluid flow in a precessing spheroidal shell. J. Fluid Mech. 33, 739-752.

Eloy, C., Le Gal, P. \& Le Dizès, S. 2000 Experimental study of the multipolar vortex instability. Phys. Rev. Lett. 85, 3400-3403.

Eloy, C., Le Gal, P. \& Le Dizès, S. 2003 Elliptic and triangular instabilities in rotating cylinders. J. Fluid Mech. 476, 357-388.

Fukumoto, Y. 2003 The three dimensional instability of a strained vortex tube revisited. $J$. Fluid Mech. 493, 287-318.

Gans, R. F. 1970a On hydromagnetic precession in a cylinder. J. Fluid Mech. 45, 111.

Gans, R. F. $1970 b$ On the precession of a resonant cylinder. J. Fluid Mech. 476, 865-872.

Gans, R. F. 1984 Dynamics of a near-resonant fluid-filled gyroscope. AIAA J. 22, 1465-1471. 
Garg, S. C., Furunoto, N. \& Vanyo, J. P. 1986 Spacecraft nutational instability prediction by energy dissipation measurments. J. Guid. 9 (3), 357-361.

Greenspan, H. P. 1968 The theory of rotating fluids. Cambridge University Press.

Hollerbach, R. \& Kerswell, R. 1995 Oscillatory internal shear layers in rotating and precessing flows. J. Fluid Mech. 298, 327.

Kelvin, L. 1880 Vibrations of a columnar vortex. Phil. Mag. 10, 155-168.

Kerswell, R. R. 1993 The instability of precessing flow. Geophys. Astrophys. Fluid Dyn. 72, $107-144$.

Kerswell, R. R. 1995 On the internal shear layers spawned by the critical regions in oscillatory ekman boudary layers. J. Fluid Mech. 298, 311-325.

Kerswell, R. R. 1996 Upper bounds on the energy dissipation in turbulent precession,. $J$. Fluid Mech. 321, 335-370.

KeRswell, R. R. 1999 Secondary instabilities in rapidly rotating fluids : inertial wave breakdown. J. Fluid Mech. 382, 283-306.

Kerswell, R. R. 2002 Elliptical instability. Ann. Rev. Fluid Mech. 34, 83-113.

Kerswell, R. R. \& Barenghi, C. F. 1995 On the viscous decay rates of inertial waves in a rotating cylinder. J. Fluid Mech. 285, 203-214.

Kobine, J. J. 1995 Inertial wave dynamics in a rotating and precessing cylinder. J. Fluid Mech. 303, 233-252.

Kobine, J. J. 1996 Azimuthal flow associated with inertial wave resonance in a precessing cylinder. J. Fluid Mech. 319, 387-406.

Kudlick, M. 1966 On the transient motions in a contained rotating fluid. PhD thesis, Massachussets Institute of Technology.

Lagrange, R., Eloy, C., Nadal, F. \& Meunier, P. 2008 Instability of a fluid inside a precessing cylinder. Physics of Fluids. 20(8), 081701.

Lambelin, J. P., Nadal, F., Lagrange, R. \& Sarthou, A. 2009 Non-resonant viscous theory for the stability of a fluid-filled gyroscope. J. Fluid Mech. pp. 08-S-0525.R2.

Lorenzani, S. \& Tilgner, A. 2001 Fluid instabilities in precessing spheroidal cavities. J. Fluid Mech. 447, 111-128.

Mahalov, A. 1993 The instability of rotating fluid columns subjected to a weak external coriolis-force. Phys. Fluids A 5 (4), 891-900.

Malkus, W. V. R. 1968 Precession of the earth as the cause of geomagnetism. Science 160, 259-264.

Malkus, W. V. R. 1989 An experimental study of global instabilities due to tidal (elliptical) distortion of a rotating elastic cylinder. Geophys. Astrophys. Fluid Dynamics 48, 123-134.

Manasseh, R. 1992 Breakdown regimes of inertia waves in a precessing cylinder. J. Fluid Mech. 243, 261-296.

Manasseh, R. 1996 Nonlinear behaviour of contained inertia waves. J. Fluid Mech. 315, 151173.

Mason, D. M. \& Kerswell, R. R. 1999 Nonlinear evolution of the elliptical instability : an example of inertial breakdown. J. Fluid Mech. 396, 73-108.

McEwan, A. D. 1970 Inertial oscillations in a rotating fluid cylinder. J. Fluid Mech. 40 (3), 603-640.

Meunier, P., Eloy, C., Lagrange, R. \& Nadal, F. 2008 A rotating fluid cylinder subject to weak precession. J. Fluid Mech. 599, 405-440.

Meunier, P. \& Leweke, T. 2003 Analysis and minimization of errors due to high gradients in particule image velocimetry. Exp. Fluids 35, 408-421.

Moore, D. W. \& Saffman, P. G. 1975 The instability of a straight vortex filament in a strain field. Proc. R. Soc. London Ser. A 346, 413-425.

Noir, J. 2000 Écoulement d'un fluide dans une cavité en précession : approches numérique et expérimentale. Ph.D. thesis, Université Joseph Fourier, Grenoble 1.

Noir, J., Brito, D., Aldridge, K. \& Cardin, P. 2001a Experimental evidence of inertial waves in a precessing spheroidal cavity. Geophys. Res. Lett. 38, 3785-3788.

Noir, J., Cardin, P., Jault, D. \& Masson, J. P. 2003 Experimental evidence of nonlinear resonance effects between retrograde precession and the tilt-over mode within a spheroid. Geophys. J. Int. 154, 407-416. 
Noir, J., Jault, D. \& Cardin, P. $2001 b$ Numerical study of the motions within a slowly precessing sphere at low Ekman number. J. Fluid Mech. 437, 283-29.

Poincaré, H. 1910 Sur la précession des corps déformables. Bull. Astro. 27, 257-264.

RACZ, J.-P. \& ScOTt, J. F. 2007 Parametric instability in a rotating cylinder of gas subject to sinusoidal axial compression. part2. weakly nonlinear theory. J. Fluid Mech. 595, 291-321.

Saffman, P. G. 1992 Vortex dynamics. Cambridge University Press.

Stewartson, K. 1958 On the stability of a spinning top containing liquid. J. Fluid Mech. 5, $577-592$.

Thompson, R. 1970 Diurnal tides and shear instabiliies in a rotating cylinder. J. Fluid Mech. 40, 737-751.

Tilgner, A. 1999a Magnetohydrodynamic flow in precessing spherical shells. J. Fluid Mech. 379, 303-318.

Tilgner, A. $1999 b$ Non-axisymmetric shear layers in precessing fluid ellipsoidal shells. Geophys. J. Int. 136, 629-636.

Tilgner, A. 2005 Precession driven dynamos. Phys. Fluids 17, 034104.

Tilgner, A. 2007 Kinematic dynamos with precession driven flow in a sphere. Geophysical and astrophysical fluid dynamics 101, 1-9.

Tsai, C.-Y. \& Widnall, S. E. 1976 The stability of short waves on a straight vortex filament in a weak externally imposed strain field. J. Fluid Mech. 73 (4), 721-733.

Vanyo, J. P., Wilde, P. \& Cardin, P. 1995 Experiments on precessing flows in the earth's liquid core. Geophys. J. Int. 121, 136-142.

WALEFFE, F. 1989 The 3d instability of a strained vortex and its relation to turbulence. $\mathrm{PhD}$ thesis, Massachusetts Institute of Technology.

Waleffe, F. 1990 On the three-dimensional instability of strained vortices. Phys. Fluids A $2(1), 76-80$.

Wu, C. \& Roberts, P. 2008 A precessionally-driven dynamo in a plane layer. Geophysical and astrophysical fluid dynamics 102, 1-19.

Wu, C. \& Roberts, P. 2009 On a dynamo driven by topographic precession. Geophysical and astrophysical fluid dynamics 103, 467-501. 